\title{
On a Hardy-Hilbert-type inequality with parameters
}

Bicheng Yang ${ }^{1 *}$ and Qiang Chen ${ }^{2}$

"Correspondence:

bcyang@gdei.edu.cn

'Department of Mathematics, Guangdong University of

Education, Guangzhou, Guangdong

510303, P.R. China

Full list of author information is

available at the end of the article

\section{Abstract}

By means of the way of weight coefficients and technique of real analysis, an extension of a Hardy-Hilbert-type inequality with parameters and a best possible constant factor is given. The equivalent forms, the operator expression with the norm, the reverses and some particular cases are also considered.

MSC: 26D15; 47A07

Keywords: Hardy-Hilbert-type inequality; weight coefficient; equivalent form; reverse; operator

\section{Introduction}

Suppose that $p>1, \frac{1}{p}+\frac{1}{q}=1, f(x), g(y) \geq 0, f \in L^{p}\left(\mathbf{R}_{+}\right), g \in L^{q}\left(\mathbf{R}_{+}\right),\|f\|_{p}=\left(\int_{0}^{\infty} f^{p}(x) d x\right)^{\frac{1}{p}}>$ $0,\|g\|_{q}>0$. We have the following Hardy-Hilbert's integral inequality with the best possible constant factor $\frac{\pi}{\sin (\pi / p)}(c f .[1])$ :

$$
\int_{0}^{\infty} \int_{0}^{\infty} \frac{f(x) g(y)}{x+y} d x d y<\frac{\pi}{\sin (\pi / p)}\|f\|_{p}\|g\|_{q}
$$

Assuming that $a_{m}, b_{n} \geq 0$,

$$
a=\left\{a_{m}\right\}_{m=1}^{\infty} \in l^{p}=\left\{a ;\|a\|_{p}=\left(\sum_{m=1}^{\infty}\left|a_{m}\right|^{p}\right)^{\frac{1}{p}}<\infty\right\},
$$

$b=\left\{b_{n}\right\}_{n=1}^{\infty} \in l^{q},\|a\|_{p},\|b\|_{q}>0$, we have the following Hardy-Hilbert's inequality with the same best possible constant factor $\frac{\pi}{\sin (\pi / p)}(c f .[1])$ :

$$
\sum_{m=1}^{\infty} \sum_{n=1}^{\infty} \frac{a_{m} b_{n}}{m+n}<\frac{\pi}{\sin (\pi / p)}\|a\|_{p}\|b\|_{q}
$$

Hardy-Hilbert-type inequalities, specially (1) and (2), are basically important in mathematical analysis and its applications (cf. [1-7]).

$$
\text { If } \begin{aligned}
\mu_{i}, v_{j} & >0(i, j \in \mathbf{N}), \\
U_{m} & :=\sum_{i=1}^{m} \mu_{i}, \quad V_{n}:=\sum_{j=1}^{n} v_{j} \quad(m, n \in \mathbf{N}),
\end{aligned}
$$

(c) 2015 Yang and Chen. This article is distributed under the terms of the Creative Commons Attribution 4.0 International License (http://creativecommons.org/licenses/by/4.0/), which permits unrestricted use, distribution, and reproduction in any medium, provided you give appropriate credit to the original author(s) and the source, provide a link to the Creative Commons license, and indicate if changes were made. 
then we have the following inequality (cf. [1], Theorem 321, p.261):

$$
\sum_{m=1}^{\infty} \sum_{n=1}^{\infty} \frac{\mu_{m}^{1 / q} v_{n}^{1 / p} a_{m} b_{n}}{U_{m}+V_{n}}<\frac{\pi}{\sin (\pi / p)}\|a\|_{p}\|b\|_{q}
$$

Replacing $\mu_{m}^{1 / q} a_{m}$ and $v_{n}^{1 / p} b_{n}$ by $a_{m}$ and $b_{n}$ in (4), respectively, we obtain the following equivalent form of (4):

$$
\sum_{m=1}^{\infty} \sum_{n=1}^{\infty} \frac{a_{m} b_{n}}{U_{m}+V_{n}}<\frac{\pi}{\sin \left(\frac{\pi}{p}\right)}\left(\sum_{m=1}^{\infty} \frac{a_{m}^{p}}{\mu_{m}^{p-1}}\right)^{\frac{1}{p}}\left(\sum_{n=1}^{\infty} \frac{b_{n}^{q}}{v_{n}^{q-1}}\right)^{\frac{1}{q}} .
$$

For $\mu_{i}=v_{j}=1(i, j \in \mathbf{N})$, both (4) and (5) reduce to (2). We call (4) and (5) Hardy-Hilberttype inequalities.

Note The authors of [1] (Theorem 321, p.261) did not prove that (4) is valid with the best possible constant factor.

In 1998, by introducing an independent parameter $\lambda \in(0,1]$, Yang [8] gave an extension of (1) for $p=q=2$. Following the methods of [8], Yang [5] gave some best extensions of (1) and (2) as follows.

If $\lambda_{1}, \lambda_{2} \in \mathbf{R}=(-\infty, \infty), \lambda_{1}+\lambda_{2}=\lambda, k_{\lambda}(x, y)$ is a nonnegative homogeneous function of degree $-\lambda$, with $k\left(\lambda_{1}\right)=\int_{0}^{\infty} k_{\lambda}(t, 1) t^{\lambda_{1}-1} d t \in \mathbf{R}_{+}, \phi(x)=x^{p\left(1-\lambda_{1}\right)-1}, \psi(x)=x^{q\left(1-\lambda_{2}\right)-1}$, $f(x), g(y) \geq 0$,

$$
f \in L_{p, \phi}\left(\mathbf{R}_{+}\right)=\left\{f ;\|f\|_{p, \phi}:=\left(\int_{0}^{\infty} \phi(x)|f(x)|^{p} d x\right)^{\frac{1}{p}}<\infty\right\}
$$

$g \in L_{q, \psi}\left(\mathbf{R}_{+}\right),\|f\|_{p, \phi},\|g\|_{q, \psi}>0$, then

$$
\int_{0}^{\infty} \int_{0}^{\infty} k_{\lambda}(x, y) f(x) g(y) d x d y<k\left(\lambda_{1}\right)\|f\|_{p, \phi}\|g\|_{q, \psi},
$$

where the constant factor $k\left(\lambda_{1}\right)$ is the best possible. Moreover, if $k_{\lambda}(x, y)$ is finite and $k_{\lambda}(x, y) x^{\lambda_{1}-1}\left(k_{\lambda}(x, y) y^{\lambda_{2}-1}\right)$ is decreasing with respect to $x>0(y>0)$, then for $a_{m}, b_{n} \geq 0$,

$$
a \in l_{p, \phi}=\left\{a ;\|a\|_{p, \phi}:=\left(\sum_{n=1}^{\infty} \phi(n)\left|a_{n}\right|^{p}\right)^{\frac{1}{p}}<\infty\right\},
$$

$b=\left\{b_{n}\right\}_{n=1}^{\infty} \in l_{q, \psi},\|a\|_{p, \phi},\|b\|_{q, \psi}>0$, we have

$$
\sum_{m=1}^{\infty} \sum_{n=1}^{\infty} k_{\lambda}(m, n) a_{m} b_{n}<k\left(\lambda_{1}\right)\|a\|_{p, \phi}\|b\|_{q, \psi}
$$

where the constant factor $k\left(\lambda_{1}\right)$ is still the best possible.

Clearly, for $\lambda=1, k_{1}(x, y)=\frac{1}{x+y}, \lambda_{1}=\frac{1}{q}, \lambda_{2}=\frac{1}{p}$, inequality (6) reduces to (1), while (7) reduces to (2). For $0<\lambda_{1}, \lambda_{2} \leq 1, \lambda_{1}+\lambda_{2}=\lambda$, we set $k_{\lambda}(x, y)=\frac{1}{(x+y)^{2}}$. Then, by (7), it follows 
that

$$
\sum_{m=1}^{\infty} \sum_{n=1}^{\infty} \frac{a_{m} b_{n}}{(m+n)^{\lambda}}<B\left(\lambda_{1}, \lambda_{2}\right)\|a\|_{p, \phi}\|b\|_{q, \psi}
$$

where the constant factor $B\left(\lambda_{1}, \lambda_{2}\right)$ is the best possible $(B(u, v)$ is the beta function). Some other results including multidimensional Hilbert-type inequalities are provided by [9-27].

In 2015, by adding a few conditions, Yang [28] gave an extension of (8) and (5) as follows:

$$
\begin{aligned}
& \sum_{m=1}^{\infty} \sum_{n=1}^{\infty} \frac{a_{m} b_{n}}{\left(U_{m}+V_{n}\right)^{\lambda}} \\
& \quad<B\left(\lambda_{1}, \lambda_{2}\right)\left(\sum_{m=1}^{\infty} \frac{U_{m}^{p\left(1-\lambda_{1}\right)-1} a_{m}^{p}}{\mu_{m}^{p-1}}\right)^{\frac{1}{p}}\left(\sum_{n=1}^{\infty} \frac{V_{n}^{q\left(1-\lambda_{2}\right)-1} b_{n}^{q}}{v_{n}^{q-1}}\right)^{\frac{1}{q}},
\end{aligned}
$$

where the constant factor $B\left(\lambda_{1}, \lambda_{2}\right)$ is the best possible. For $\mu_{i}=v_{j}=1(i, j \in \mathbf{N}),(9)$ reduces to (8); for $\lambda=1, \lambda_{1}=\frac{1}{q}, \lambda_{2}=\frac{1}{p}$, (9) reduces to (5).

In this paper, by using the way of weight coefficients and technique of real analysis, a Hardy-Hilbert-type inequality with parameters and a best possible constant factor is given, which is with the kernel $\frac{\left(\min \left\{x, c_{1} y\right\}\right)^{\alpha}}{\left(\max \left\{x, c_{1} y\right\}\right)^{\lambda+\alpha}}$ similar to (9). The extended inequalities, the equivalent forms, the operator expression with the norm, the reverses and some particular cases are also considered.

\section{Some lemmas}

In the following, we agree on that $\mu_{i}, v_{j}>0(i, j \in \mathbf{N}), U_{m}$ and $V_{n}$ are defined by (3), $p \neq 0,1$, $\frac{1}{p}+\frac{1}{q}=1, a_{m}, b_{n} \geq 0(m, n \in \mathbf{N}),\|a\|_{p, \Phi_{\lambda}}=\left(\sum_{m=1}^{\infty} \Phi_{\lambda}(m) a_{m}^{p}\right)^{\frac{1}{p}},\|b\|_{q, \Psi_{\lambda}}=\left(\sum_{n=1}^{\infty} \Psi_{\lambda}(n) b_{n}^{q}\right)^{\frac{1}{q}}$, where

$$
\Phi_{\lambda}(m):=\frac{U_{m}^{p\left(1-\lambda_{1}\right)-1}}{\mu_{m}^{p-1}}, \quad \Psi_{\lambda}(n):=\frac{V_{n}^{q\left(1-\lambda_{2}\right)-1}}{v_{n}^{q-1}} \quad(m, n \in \mathbf{N}) .
$$

Lemma 1 If $g(t)(>0)$ is decreasing in $\mathbf{R}_{+}$and strictly decreasing in $\left[n_{0}, \infty\right) \subset \mathbf{R}_{+}\left(n_{0} \in \mathbf{N}\right)$, satisfying $\int_{0}^{\infty} g(t) d t \in \mathbf{R}_{+}$, then we have

$$
\int_{1}^{\infty} g(t) d t<\sum_{n=1}^{\infty} g(n)<\int_{0}^{\infty} g(t) d t
$$

Proof Since, by the assumption, we have

$$
\begin{aligned}
& \int_{n}^{n+1} g(t) d t \leq g(n) \leq \int_{n-1}^{n} g(t) d t \quad\left(n=1, \ldots, n_{0}\right), \\
& \int_{n_{0}+1}^{n_{0}+2} g(t) d t<g\left(n_{0}+1\right)<\int_{n_{0}}^{n_{0}+1} g(t) d t
\end{aligned}
$$

it follows that

$$
0<\int_{1}^{n_{0}+2} g(t) d t<\sum_{n=1}^{n_{0}+1} g(n)<\sum_{n=1}^{n_{0}+1} \int_{n-1}^{n} g(t) d t=\int_{0}^{n_{0}+1} g(t) d t<\infty .
$$


By the same way, we still have

$$
0<\int_{n_{0}+2}^{\infty} g(t) d t \leq \sum_{n=n_{0}+2}^{\infty} g(n) \leq \int_{n_{0}+1}^{\infty} g(t) d t<\infty
$$

Hence, making plus for the above two inequalities, we have (10).

Example 1 For $s \in \mathbf{N}, 0<c_{1} \leq \cdots \leq c_{s}<\infty, \lambda_{1}, \lambda_{2}>-\alpha, \lambda_{1}+\lambda_{2}=\lambda$, we set

$$
k_{\lambda}(x, y):=\prod_{k=1}^{s} \frac{\left(\min \left\{x, c_{k} y\right\}\right)^{\frac{\alpha}{s}}}{\left(\max \left\{x, c_{k} y\right\}\right)^{\frac{\lambda+\alpha}{s}}} \quad\left((x, y) \in \mathbf{R}_{+}^{2}=\mathbf{R}_{+} \times \mathbf{R}_{+}\right) .
$$

(a) We find

$$
\begin{aligned}
& k_{s}\left(\lambda_{1}\right):=\int_{0}^{\infty} k_{\lambda}(1, u) t^{\lambda_{2}-1} d u \stackrel{u=1 / t}{=} \int_{0}^{\infty} k_{\lambda}(t, 1) t^{\lambda_{1}-1} d t \\
& =\int_{0}^{\infty} \prod_{k=1}^{s} \frac{\left(\min \left\{t, c_{k}\right\}\right)^{\frac{\alpha}{s}}}{\left(\max \left\{t, c_{k}\right\}\right)^{\frac{\lambda+\alpha}{s}}} t^{\lambda_{1}-1} d t \\
& =\int_{0}^{c_{1}} \prod_{k=1}^{s} \frac{\left(\min \left\{t, c_{k}\right\}\right)^{\frac{\alpha}{s}} t^{\lambda_{1}-1}}{\left(\max \left\{t, c_{k}\right\}\right)^{\frac{\lambda+\alpha}{s}}} d t+\int_{c_{s}}^{\infty} \prod_{k=1}^{s} \frac{\left(\min \left\{t, c_{k}\right\}\right)^{\frac{\alpha}{s}} t^{\lambda_{1}-1}}{\left(\max \left\{t, c_{k}\right\}\right)^{\frac{\lambda+\alpha}{s}}} d t \\
& +\sum_{i=1}^{s-1} \int_{c_{i}}^{c_{i+1}} \prod_{k=1}^{s} \frac{\left(\min \left\{t, c_{k}\right\}\right)^{\frac{\alpha}{s}} t^{\lambda_{1}-1}}{\left(\max \left\{t, c_{k}\right\}\right)^{\frac{\lambda+\alpha}{s}}} d t \\
& =\prod_{k=1}^{s} \frac{1}{c_{k}^{(\lambda+\alpha) / s}} \int_{0}^{c_{1}} t^{\lambda_{1}+\alpha-1} d t+\prod_{k=1}^{s} c_{k}^{\alpha / s} \int_{c_{s}}^{\infty} t^{-\lambda_{2}-\alpha-1} d t \\
& +\sum_{i=1}^{s-1} \int_{c_{i}}^{c_{i+1}} \prod_{k=1}^{i} \frac{c_{k}^{\frac{\alpha}{s}}}{t^{\frac{\lambda+\alpha}{s}}} \prod_{k=i+1}^{s} \frac{t^{\frac{\alpha}{s}}}{c_{k}^{\frac{\lambda+\alpha}{s}}} t^{\lambda_{1}-1} d t \\
& =\frac{c_{1}^{\lambda_{1}+\alpha}}{\lambda_{1}+\alpha} \frac{1}{\prod_{k=1}^{s} c_{k}^{\frac{\lambda+\alpha}{s}}}+\frac{1}{\left(\lambda_{2}+\alpha\right) c_{s}^{\lambda_{2}+\alpha}} \prod_{k=1}^{s} c_{k}^{\frac{\alpha}{s}} \\
& +\sum_{i=1}^{s-1} \frac{\prod_{k=1}^{i} c_{k}^{\frac{\alpha}{s}}}{\prod_{k=i+1}^{s} c_{k}^{\frac{\lambda+\alpha}{s}}} \int_{c_{i}}^{c_{i+1}} t^{\lambda_{1}-\frac{i \lambda}{s}+\left(1-\frac{2 i}{s}\right) \alpha-1} d t .
\end{aligned}
$$

If $\lambda_{1}-\frac{i \lambda}{s}+\left(1-\frac{2 i}{s}\right) \alpha \neq 0$, then

$$
\int_{c_{i}}^{c_{i+1}} t^{\lambda_{1}-\frac{i \lambda}{s}+\left(1-\frac{2 i}{s}\right) \alpha-1} d t=\frac{c_{i+1}^{\lambda_{1}-\frac{i \lambda}{s}+\left(1-\frac{2 i}{s}\right) \alpha}-c_{i}^{\lambda_{1}-\frac{i \lambda}{s}+\left(1-\frac{2 i}{s}\right) \alpha}}{\lambda_{1}-\frac{i \lambda}{s}+\left(1-\frac{2 i}{s}\right) \alpha}
$$

if there exists $i_{0} \in\{1, \ldots, s-1\}$ such that $\lambda_{1}-\frac{i_{0} \lambda}{s}+\left(1-\frac{2 i_{0}}{s}\right) \alpha=0$, then we find

$$
\int_{c_{i_{0}}}^{c_{i_{0}+1}} t^{\lambda_{1}-\frac{i_{0} \lambda}{s}+\left(1-\frac{2 i_{0}}{s}\right) \alpha-1} d t=\ln \left(\frac{c_{i_{0}+1}}{c_{i_{0}}}\right)=\lim _{i \rightarrow i_{0}} \int_{c_{i}}^{c_{i+1}} t^{\lambda_{1}-\frac{i \lambda}{s}+\left(1-\frac{2 i}{s}\right) \alpha-1} d t,
$$


and we still indicate $\ln \left(\frac{c_{i_{0}+1}}{c_{i_{0}}}\right)$ by the following formal expression:

$$
\frac{c_{i_{0}+1}^{\lambda_{1} \frac{i_{0} \lambda}{s}+\left(1-\frac{2 i_{0}}{s}\right) \alpha}-c_{i_{0}}^{\lambda_{1}-\frac{i_{0} \lambda}{s}+\left(1-\frac{2 i_{0}}{s}\right) \alpha}}{\lambda_{1}-\frac{i_{0} \lambda}{s}+\left(1-\frac{2 i_{0}}{s}\right) \alpha} .
$$

Hence, we may set

$$
\begin{aligned}
k_{s}\left(\lambda_{1}\right)= & \frac{c_{1}^{\lambda_{1}+\alpha}}{\lambda_{1}+\alpha} \frac{1}{\prod_{k=1}^{s} c_{k}^{\frac{\lambda+\alpha}{s}}}+\frac{1}{\left(\lambda_{2}+\alpha\right) c_{s}^{\lambda_{2}+\alpha}} \prod_{k=1}^{s} c_{k}^{\frac{\alpha}{s}} \\
& +\sum_{i=1}^{s-1}\left[\frac{c_{i+1}^{\lambda_{1}-\frac{i \lambda}{s}+\left(1-\frac{2 i}{s}\right) \alpha}-c_{i}^{\lambda_{1}-\frac{i \lambda}{s}+\left(1-\frac{2 i}{s}\right) \alpha}}{\lambda_{1}-\frac{i \lambda}{s}+\left(1-\frac{2 i}{s}\right) \alpha} \frac{\prod_{k=1}^{i} c_{k}^{\frac{\alpha}{s}}}{\prod_{k=i+1}^{s} c_{k}^{\frac{\lambda+\alpha}{s}}}\right] .
\end{aligned}
$$

In particular, (i) for $s=1\left(\right.$ or $\left.c_{s}=\cdots=c_{1}\right)$, we have $k_{\lambda}(x, y)=\frac{\left(\min \left\{x, c_{1} y\right)^{\alpha}\right.}{\left(\max \left\{x, c_{1} y\right\}\right)^{\lambda+\alpha}}$ and

$$
k_{1}\left(\lambda_{1}\right)=\frac{\lambda+2 \alpha}{\left(\lambda_{1}+\alpha\right)\left(\lambda_{2}+\alpha\right)} \frac{1}{c_{1}^{\lambda_{2}}}
$$

(ii) for $s=2$, we have $k_{\lambda}(x, y)=\frac{\left(\min \left\{x, c_{1} y\right\} \min \left\{x, c_{2} y\right\}\right)^{\alpha / 2}}{\left(\max \left\{x, c_{1} y\right\} \max \left\{x, c_{2} y\right\}\right)^{(\lambda+\alpha) / 2}}$ and

$$
k_{2}\left(\lambda_{1}\right)=\left(\frac{c_{1}}{c_{2}}\right)^{\frac{\alpha}{2}}\left[\frac{c_{1}^{\lambda_{1}-\frac{\lambda}{2}}}{\left(\lambda_{1}+\alpha\right) c_{2}^{\frac{\lambda}{2}}}+\frac{1}{\left(\lambda_{2}+\alpha\right) c_{2}^{\lambda_{2}}}+\frac{c_{2}^{\lambda_{1}-\frac{\lambda}{2}}-c_{1}^{\lambda_{1}-\frac{\lambda}{2}}}{\left(\lambda_{1}-\frac{\lambda}{2}\right) c_{2}^{\frac{\lambda}{2}}}\right]
$$

(iii) for $\alpha=0$, we have $\lambda_{1}, \lambda_{2}>0, k_{\lambda}(x, y)=\frac{1}{\prod_{k=1}^{s}\left(\max \left\{x, c_{k} y\right\}\right)^{\frac{\lambda}{s}}}$ and

$$
k_{s}\left(\lambda_{1}\right)=\widetilde{k}_{s}\left(\lambda_{1}\right):=\frac{c_{1}^{\lambda_{1}}}{\lambda_{1}} \frac{1}{\prod_{k=1}^{s} c_{k}^{\frac{\lambda}{s}}}+\frac{1}{\lambda_{2} c_{s}^{\lambda_{2}}}+\sum_{i=1}^{s-1} \frac{c_{i+1}^{\lambda_{1}-\frac{i}{s} \lambda}-c_{i}^{\lambda_{1}-\frac{i}{s} \lambda}}{\lambda_{1}-\frac{i}{s} \lambda} \frac{1}{\prod_{k=i+1}^{s} c_{k}^{\frac{\lambda}{s}}} ;
$$

(iv) for $\alpha=-\lambda$, we have $\lambda<\lambda_{1}, \lambda_{2}<0, k_{\lambda}(x, y)=\frac{1}{\prod_{k=1}^{s}\left(\min \left\{x, c_{k} y\right\}\right)^{\frac{\lambda}{s}}}$ and

$$
k_{s}\left(\lambda_{1}\right)=\widehat{k}_{s}\left(\lambda_{1}\right):=\frac{c_{1}^{-\lambda_{2}}}{\left(-\lambda_{2}\right)}+\frac{1}{\left(-\lambda_{1}\right) c_{s}^{-\lambda_{1}}} \prod_{k=1}^{s} c_{k}^{\frac{-\lambda}{s}}+\sum_{i=1}^{s-1}\left(\frac{c_{i+1}^{\lambda_{1}-\frac{s-i}{s} \lambda}-c_{i}^{\lambda_{1}-\frac{s-i}{s}}}{\lambda_{1}-\frac{s-i}{s} \lambda} \prod_{k=1}^{i} c_{k}^{\frac{-\lambda}{s}}\right) ;
$$

(v) for $\lambda=0$, we have $\lambda_{2}=-\lambda_{1},\left|\lambda_{1}\right|<\alpha(\alpha>0)$,

$$
k_{0}(x, y)=\prod_{k=1}^{s}\left(\frac{\min \left\{x, c_{k} y\right\}}{\max \left\{x, c_{k} y\right\}}\right)^{\frac{\alpha}{s}}
$$

and

$$
\begin{aligned}
k_{s}\left(\lambda_{1}\right)= & k_{s}^{(0)}\left(\lambda_{1}\right):=\frac{c_{1}^{\lambda_{1}+\alpha}}{a+\lambda_{1}} \frac{1}{\prod_{k=1}^{s} c_{k}^{\frac{\alpha}{s}}}+\frac{c_{s}^{\lambda_{1}-\alpha}}{a-\lambda_{1}} \prod_{k=1}^{s} c_{k}^{\frac{\alpha}{s}} \\
& +\sum_{i=1}^{s-1}\left[\frac{c_{i+1}^{\lambda_{1}+\left(1-\frac{2 i}{s}\right) \alpha}-c_{i}^{\lambda_{1}+\left(1-\frac{2 i}{s}\right) \alpha}}{\lambda_{1}+\left(1-\frac{2 i}{s}\right) \alpha} \frac{\prod_{k=1}^{i} c_{k}^{\frac{\alpha}{s}}}{\prod_{k=i+1}^{s} c_{k}^{\frac{\alpha}{s}}}\right] .
\end{aligned}
$$


(b) Since we find

$$
\begin{aligned}
& k_{\lambda}(x, y) \frac{1}{y^{1-\lambda_{2}}}=\frac{1}{y^{1-\lambda_{2}}} \prod_{k=1}^{s} \frac{\left(\min \left\{c_{k}^{-1} x, y\right\}\right)^{\frac{\alpha}{s}}}{c_{k}^{\frac{\lambda}{s}}\left(\max \left\{c_{k}^{-1} x, y\right\}\right)^{\frac{\lambda+\alpha}{s}}} \\
& = \begin{cases}\frac{1}{y^{1-\lambda \lambda_{2}-\alpha}} \prod_{k=1}^{s} \frac{1}{c_{k}^{\frac{\lambda}{s}}\left(c_{k}^{-1} x\right)^{\frac{\lambda+\alpha}{s}}}, & 0<y \leq c_{s}^{-1} x, \\
\frac{1}{y^{1+\lambda 1_{1}+\alpha-\frac{i}{s}(\lambda+2 \alpha)} \frac{\prod_{k=i+1}^{s}\left(c_{k}^{-1} x\right)^{\frac{\alpha}{s}}}{\prod_{k=1}^{s} c_{k}^{\frac{\lambda}{s}} \prod_{k=1}^{i}\left(c_{k}^{-1} x\right)^{\frac{\lambda+\alpha}{s}}},} & c_{i+1}^{-1} x<y \leq c_{i}^{-1} x(i=1, \ldots, s-1), \\
\frac{1}{y^{1+\lambda 1+\alpha}} \prod_{k=1}^{s} \frac{\left(c_{k}^{-1} x\right)^{\frac{\alpha}{s}}}{c_{k}^{\frac{\lambda}{s}}(y)^{\frac{\lambda+\alpha}{s}}}, & c_{1}^{-1} x<y<\infty,\end{cases}
\end{aligned}
$$

then for $\lambda_{2} \leq 1-\alpha\left(\lambda_{1}>-\alpha\right), k_{\lambda}(x, y) \frac{1}{y^{1-\lambda_{2}}}$ is decreasing for $y>0$ and strictly decreasing for the large enough variable $y$. By the same way, since

$$
\begin{aligned}
& k_{\lambda}(x, y) \frac{1}{x^{1-\lambda_{1}}}=\frac{1}{x^{1-\lambda_{1}}} \prod_{k=1}^{s} \frac{\left(\min \left\{x, c_{k} y\right\}\right)^{\frac{\alpha}{s}}}{\left(\max \left\{x, c_{k} y\right\}\right)^{\frac{\lambda+\alpha}{s}}} \\
& = \begin{cases}\frac{1}{x^{1-\lambda} 1^{-\alpha}} \prod_{k=1}^{s} \frac{1}{\left(c_{k} y\right)^{\frac{\lambda+\alpha}{s}}}, & 0<x \leq c_{1} y, \\
\frac{1}{x^{1-\lambda} 1^{-\alpha+\frac{i}{s}(\lambda+2 \alpha)}} \frac{\prod_{k=1}^{i}\left(c_{k} y\right)^{\frac{\alpha}{s}}}{\prod_{k=i+1}^{s}\left(c_{k} y\right)^{\frac{\lambda+\alpha}{s}}}, & c_{i} y<x \leq c_{i+1} y(i=1, \ldots, s-1), \\
\frac{1}{x^{1+\lambda} 2^{2+\alpha}} \prod_{k=1}^{s}\left(c_{k} y\right)^{\frac{\alpha}{s}}, & c_{s} y<x<\infty,\end{cases}
\end{aligned}
$$

then for $\lambda_{1} \leq 1-\alpha\left(\lambda_{2}>-\alpha\right), k_{\lambda}(x, y) \frac{1}{x^{1-\lambda_{1}}}$ is decreasing for $x>0$ and strictly decreasing for the large enough variable $x$.

In view of (a) and (b), for $-\alpha<\lambda_{1}, \lambda_{2} \leq 1-\alpha, \lambda_{1}+\lambda_{2}=\lambda, k_{\lambda}(x, y) \frac{1}{y^{1-\lambda_{2}}}\left(k_{\lambda}(x, y) \frac{1}{x^{1-\lambda_{1}}}\right)$ is decreasing for $y>0(x>0)$ and strictly decreasing for the large enough variable $y(x)$ satisfying $k_{s}\left(\lambda_{1}\right) \in \mathbf{R}_{+}$.

Lemma 2 If $s \in \mathbf{N}, 0<c_{1} \leq \cdots \leq c_{s},-\alpha<\lambda_{1}, \lambda_{2} \leq 1-\alpha, \lambda_{1}+\lambda_{2}=\lambda, k_{s}\left(\lambda_{1}\right)$ is indicated by (11), define the following weight coefficients:

$$
\begin{aligned}
& \omega\left(\lambda_{2}, m\right):=\sum_{n=1}^{\infty} \prod_{k=1}^{s} \frac{\left(\min \left\{U_{m}, c_{k} V_{n}\right\}\right)^{\frac{\alpha}{s}}}{\left(\max \left\{U_{m}, c_{k} V_{n}\right\}\right)^{\frac{\lambda+\alpha}{s}}} \frac{U_{m}^{\lambda_{1}} v_{n}}{V_{n}^{1-\lambda_{2}}}, \quad m \in \mathbf{N}, \\
& \varpi\left(\lambda_{1}, n\right):=\sum_{m=1}^{\infty} \prod_{k=1}^{s} \frac{\left(\min \left\{U_{m}, c_{k} V_{n}\right\}\right)^{\frac{\alpha}{s}}}{\left(\max \left\{U_{m}, c_{k} V_{n}\right\}\right)^{\frac{\lambda+\alpha}{s}}} \frac{V_{n}^{\lambda_{2}} \mu_{m}}{U_{m}^{1-\lambda_{1}}}, \quad n \in \mathbf{N} .
\end{aligned}
$$

Then we have the following inequalities:

$$
\begin{array}{ll}
\omega\left(\lambda_{2}, m\right)<k_{s}\left(\lambda_{1}\right) & \left(-\alpha<\lambda_{2} \leq 1-\alpha, \lambda_{1}>-\alpha ; m \in \mathbf{N}\right), \\
\varpi\left(\lambda_{1}, n\right)<k_{s}\left(\lambda_{1}\right) & \left(-\alpha<\lambda_{1} \leq 1-\alpha, \lambda_{2}>-\alpha ; n \in \mathbf{N}\right) .
\end{array}
$$

Proof We set $\mu(t):=\mu_{m}, t \in(m-1, m](m \in \mathbf{N}) ; v(t):=v_{n}, t \in(n-1, n](n \in \mathbf{N})$,

$$
U(x):=\int_{0}^{x} \mu(t) d t \quad(x \geq 0), \quad V(y):=\int_{0}^{y} v(t) d t \quad(y \geq 0)
$$


Then, by (3), it follows that $U(m)=U_{m}, V(n)=V_{n}(m, n \in \mathbf{N})$. For $x \in(m-1, m], U^{\prime}(x)=$ $\mu(x)=\mu_{m}(m \in \mathbf{N})$; for $y \in(n-1, n], V^{\prime}(y)=v(y)=v_{n}(n \in \mathbf{N})$. Since $V(y)$ is strictly increasing in $(n-1, n],-\alpha<\lambda_{2} \leq 1-\alpha, \lambda_{1}>-\alpha$, in view of Lemma 1 and Example 1 , we find

$$
\begin{aligned}
\omega\left(\lambda_{2}, m\right) & =\sum_{n=1}^{\infty} \int_{n-1}^{n} \prod_{k=1}^{s} \frac{\left(\min \left\{U_{m}, c_{k} V_{n}\right\}\right)^{\frac{\alpha}{s}}}{\left(\max \left\{U_{m}, c_{k} V_{n}\right\}\right)^{\frac{\lambda+\alpha}{s}}} \frac{U_{m}^{\lambda_{1}}}{V_{n}^{1-\lambda_{2}}} V^{\prime}(y) d y \\
& <\sum_{n=1}^{\infty} \int_{n-1}^{n} \prod_{k=1}^{s} \frac{\left(\min \left\{U_{m}, c_{k} V(y)\right\}\right)^{\frac{\alpha}{s}}}{\left(\max \left\{U_{m}, c_{k} V(y)\right\}\right)^{\frac{\lambda+\alpha}{s}}} \frac{U_{m}^{\lambda_{1}} V^{\prime}(y)}{V^{1-\lambda_{2}}(y)} d y .
\end{aligned}
$$

Setting $t=\frac{V(y)}{U_{m}}$, we obtain $V^{\prime}(y) d y=U_{m} d t$ and

$$
\begin{aligned}
\omega\left(\lambda_{2}, m\right) & <\sum_{n=1}^{\infty} \int_{\frac{V(n-1)}{U_{m}}}^{\frac{V(n)}{U_{m}}} \prod_{k=1}^{s} \frac{\left(\min \left\{1, c_{k} t\right\}\right)^{\frac{\alpha}{s}}}{\left(\max \left\{1, c_{k} t\right\}\right)^{\frac{\lambda+\alpha}{s}}} t^{\lambda_{2}-1} d t \\
& =\int_{0}^{\frac{V(\infty)}{u_{m}}} \prod_{k=1}^{s} \frac{\left(\min \left\{1, c_{k} t\right\}\right)^{\frac{\alpha}{s}}}{\left(\max \left\{1, c_{k} t\right\}\right)^{\frac{\lambda+\alpha}{s}}} t^{\lambda_{2}-1} d t \\
& \leq \int_{0}^{\infty} \prod_{k=1}^{s} \frac{\left(\min \left\{1, c_{k} t\right\}\right)^{\frac{\alpha}{s}}}{\left(\max \left\{1, c_{k} t\right\}\right)^{\frac{\lambda+\alpha}{s}}} t^{\lambda_{2}-1} d t=k_{s}\left(\lambda_{1}\right) .
\end{aligned}
$$

Since $U(x)$ is strictly increasing in $(m-1, m],-\alpha<\lambda_{1} \leq 1-\alpha, \lambda_{2}>-\alpha$, by the same way, we have

$$
\begin{aligned}
\varpi\left(\lambda_{1}, n\right) & =\sum_{m=1}^{\infty} \int_{m-1}^{m} \prod_{k=1}^{s} \frac{\left(\min \left\{U_{m}, c_{k} V_{n}\right\}\right)^{\frac{\alpha}{s}}}{\left(\max \left\{U_{m}, c_{k} V_{n}\right\}\right)^{\frac{\lambda+\alpha}{s}}} \frac{V_{n}^{\lambda_{2}} U^{\prime}(x)}{U_{m}^{1-\lambda_{1}}} d x \\
& <\sum_{m=1}^{\infty} \int_{m-1}^{m} \prod_{k=1}^{s} \frac{\left(\min \left\{U(x), c_{k} V_{n}\right\}\right)^{\frac{\alpha}{s}}}{\left(\max \left\{U(x), c_{k} V_{n}\right\}\right)^{\frac{\lambda+\alpha}{s}}} \frac{V_{n}^{\lambda_{2}} U^{\prime}(x)}{U^{1-\lambda_{1}(x)}} d x \\
t=U \stackrel{U(x) / V_{n}}{=} & \sum_{m=1}^{\infty} \int_{\frac{U(m-1)}{V_{n}}}^{\frac{U(m)}{V_{n}}} \prod_{k=1}^{s} \frac{\left(\min \left\{t, c_{k}\right\}\right)^{\frac{\alpha}{s}}}{\left(\max \left\{t, c_{k}\right\}\right)^{\frac{\lambda+\alpha}{s}}} t^{\lambda_{1}-1} d t \\
= & \int_{0}^{\frac{U(\infty)}{V_{n}}} \prod_{k=1}^{s} \frac{\left(\min \left\{t, c_{k}\right\}\right)^{\frac{\alpha}{s}}}{\left(\max \left\{t, c_{k}\right\}\right)^{\frac{\lambda+\alpha}{s}} t^{\lambda_{1}-1} d t \leq k_{s}\left(\lambda_{1}\right) .}
\end{aligned}
$$

Hence, we have (19) and (20).

Lemma 3 If $s \in \mathbf{N}, 0<c_{1} \leq \cdots \leq c_{s},-\alpha<\lambda_{1}, \lambda_{2} \leq 1-\alpha, \lambda_{1}+\lambda_{2}=\lambda, k_{s}\left(\lambda_{1}\right)$ is indicated by (11), $m_{0}, n_{0} \in \mathbf{N}, \mu_{m} \geq \mu_{m+1}\left(m \in\left\{m_{0}, m_{0}+1, \ldots\right\}\right), v_{n} \geq v_{n+1}\left(n \in\left\{n_{0}, n_{0}+1, \ldots\right\}\right), U(\infty)=$ $V(\infty)=\infty$, then (i) for $m, n \in \mathbf{N}$, we have

$$
\begin{aligned}
& k_{s}\left(\lambda_{1}\right)\left(1-\theta\left(\lambda_{2}, m\right)\right)<\omega\left(\lambda_{2}, m\right) \quad\left(-\alpha<\lambda_{2} \leq 1-\alpha, \lambda_{1}>-\alpha\right), \\
& k_{s}\left(\lambda_{1}\right)\left(1-\vartheta\left(\lambda_{1}, n\right)\right)<\varpi\left(\lambda_{1}, n\right) \quad\left(-\alpha<\lambda_{1} \leq 1-\alpha, \lambda_{2}>-\alpha\right),
\end{aligned}
$$


where

$$
\begin{aligned}
& \theta\left(\lambda_{2}, m\right):=\frac{1}{k_{s}\left(\lambda_{1}\right)} \int_{0}^{\frac{U_{m_{0}}}{V_{n}}} \prod_{k=1}^{s} \frac{\left(\min \left\{t, c_{k}\right\}\right)^{\frac{\alpha}{s}}}{\left(\max \left\{t, c_{k}\right\}\right)^{\frac{\lambda+\alpha}{s}}} t^{\lambda_{1}-1} d t=O\left(\frac{1}{U_{m}^{\lambda_{2}+\alpha}}\right) \in(0,1), \\
& \vartheta\left(\lambda_{1}, n\right):=\frac{1}{k_{s}\left(\lambda_{1}\right)} \int_{0}^{\frac{U_{m_{0}}}{V_{n}}} \prod_{k=1}^{s} \frac{\left(\min \left\{t, c_{k}\right\}\right)^{\frac{\alpha}{s}}}{\left(\max \left\{t, c_{k}\right\}\right)^{\frac{\lambda+\alpha}{s}}} t^{\lambda_{1}-1} d t=O\left(\frac{1}{V_{n}^{\lambda_{1}+\alpha}}\right) \in(0,1) ;
\end{aligned}
$$

(ii) for any $b>0$, we have

$$
\begin{aligned}
& \sum_{m=1}^{\infty} \frac{\mu_{m}}{U_{m}^{1+b}}=\frac{1}{b}\left(\frac{1}{U_{m_{0}}^{b}}+b O(1)\right), \\
& \sum_{n=1}^{\infty} \frac{v_{n}}{V_{n}^{1+b}}=\frac{1}{b}\left(\frac{1}{V_{n_{0}}^{b}}+b \widetilde{O}(1)\right) .
\end{aligned}
$$

Proof Since $v_{n} \geq v_{n+1}\left(n \geq n_{0}\right),-\alpha<\lambda_{2} \leq 1-\alpha, \lambda_{1}>-\alpha$ and $V(\infty)=\infty$, by Lemma 1 , we have

$$
\begin{aligned}
\omega\left(\lambda_{2}, m\right) & \geq \sum_{n=n_{0}}^{\infty} \prod_{k=1}^{s} \frac{\left(\min \left\{U_{m}, c_{k} V_{n}\right\}\right)^{\frac{\alpha}{s}}}{\left(\max \left\{U_{m}, c_{k} V_{n}\right\}\right)^{\frac{\lambda+\alpha}{s}}} \frac{U_{m}^{\lambda_{1}}}{V_{n}^{1-\lambda_{2}}} v_{n+1} \\
& =\sum_{n=n_{0}}^{\infty} \int_{n}^{n+1} \prod_{k=1}^{s} \frac{\left(\left\{U_{m}, c_{k} V_{n}\right\}\right)^{\frac{\alpha}{s}}}{\left(\max \left\{U_{m}, c_{k} V_{n}\right\}\right)^{\frac{\lambda+\alpha}{s}}} \frac{U_{m}^{\lambda_{1}} V^{\prime}(y)}{V_{n}^{1-\lambda_{2}}} d y \\
& >\sum_{n=n_{0}}^{\infty} \int_{n}^{n+1} \prod_{k=1}^{s} \frac{\left(\left\{U_{m}, c_{k} V(y)\right\}\right)^{\frac{\alpha}{s}}}{\left(\max \left\{U_{m}, c_{k} V(y)\right\}\right)^{\frac{\lambda+\alpha}{s}}} \frac{U_{m}^{\lambda_{1}} V^{\prime}(y)}{V^{1-\lambda_{2}}(y)} d y \\
& =\sum_{n=n_{0}}^{\infty} \int_{\frac{V(n)}{U_{m}}}^{\frac{V(n+1)}{U_{m}}} \prod_{k=1}^{s} \frac{\left(\min \left\{1, c_{k} t\right\}\right)^{\frac{\alpha}{s}}}{\left(\max \left\{1, c_{k} t\right\}\right)^{\frac{\lambda+\alpha}{s}}} t^{\lambda_{2}-1} d t \\
& =\int_{\frac{V_{n}}{U_{m}}}^{\infty} \prod_{k=1}^{s} \frac{\left(\min \left\{1, c_{k} t\right\}\right)^{\frac{\alpha}{s}} t^{\lambda_{2}-1}}{\left(\max \left\{1, c_{k} t\right\}\right)^{\frac{\lambda+\alpha}{s}}} d t=k_{s}\left(\lambda_{1}\right)\left(1-\theta\left(\lambda_{2}, m\right)\right) .
\end{aligned}
$$

For $U_{m}>c_{s} V_{n_{0}}$, we obtain $c_{k} t \leq c_{s} t \leq c_{s} \frac{V_{n_{0}}}{U_{m}}<1\left(t \in\left(0, \frac{V_{n_{0}}}{U_{m}}\right] ; k=1, \ldots, s\right)$ and

$$
\theta\left(\lambda_{2}, m\right)=\frac{\prod_{k=1}^{s} c_{k}}{k_{s}\left(\lambda_{1}\right)} \int_{0}^{\frac{V_{n_{0}}}{U_{m}}} t^{\lambda_{2}+\alpha-1} d t=\frac{\prod_{k=1}^{s} c_{k}}{\left(\lambda_{2}+\alpha\right) k_{s}\left(\lambda_{1}\right)}\left(\frac{V_{n_{0}}}{U_{m}}\right)^{\lambda_{2}+\alpha},
$$

and then $\theta\left(\lambda_{2}, m\right)=O\left(\frac{1}{U_{m}^{\lambda_{2}+\alpha}}\right)$. Hence we have (22).

By the same way, since $\mu_{m} \geq \mu_{m+1}\left(m \geq m_{0}\right),-\alpha<\lambda_{1} \leq 1-\alpha, \lambda_{2}>-\alpha$ and $U(\infty)=\infty$, we have

$$
\begin{aligned}
\varpi\left(\lambda_{1}, n\right) & \geq \sum_{m=m_{0}}^{\infty} \prod_{k=1}^{s} \frac{\left(\min \left\{U_{m}, c_{k} V_{n}\right\}\right)^{\frac{\alpha}{s}}}{\left(\max \left\{U_{m}, c_{k} V_{n}\right\}\right)^{\frac{\lambda+\alpha}{s}}} \frac{V_{n}^{\lambda_{2}} \mu_{m+1}}{U_{m}^{1-\lambda_{1}}} \\
& =\sum_{m=m_{0}}^{\infty} \int_{m}^{m+1} \prod_{k=1}^{s} \frac{\left(\min \left\{U_{m}, c_{k} V_{n}\right\}\right)^{\frac{\alpha}{s}}}{\left(\max \left\{U_{m}, c_{k} V_{n}\right\}\right)^{\frac{\lambda+\alpha}{s}}} \frac{V_{n}^{\lambda_{2}} U^{\prime}(x)}{U_{m}^{1-\lambda_{1}}} d x
\end{aligned}
$$




$$
\begin{gathered}
>\quad \sum_{m=m_{0}}^{\infty} \int_{m}^{m+1} \prod_{k=1}^{s} \frac{\left(\min \left\{U(x), c_{k} V_{n}\right\}\right)^{\frac{\alpha}{s}}}{\left(\max \left\{U(x), c_{k} V_{n}\right\}\right)^{\frac{\lambda+\alpha}{s}}} \frac{V_{n}^{\lambda_{2}} U^{\prime}(x)}{U^{1-\lambda_{1}}(x)} d x \\
\stackrel{t=U(x) / V_{n}}{=} \sum_{m=m_{0}}^{\infty} \int_{\frac{U(m)}{V_{n}}}^{\frac{U(m+1)}{V_{n}}} \prod_{k=1}^{s} \frac{\left(\min \left\{t, c_{k}\right\}\right)^{\frac{\alpha}{s}}}{\left(\max \left\{t, c_{k}\right\}\right)^{\frac{\lambda+\alpha}{s}}} t^{\lambda_{1}-1} d t \\
=\quad \int_{\frac{U_{m_{0}}}{V_{n}}}^{\infty} \prod_{k=1}^{s} \frac{\left(\min \left\{t, c_{k}\right\}\right)^{\frac{\alpha}{s}} t^{\lambda_{1}-1}}{\left(\max \left\{t, c_{k}\right\}\right)^{\frac{\lambda+\alpha}{s}}} d t=k_{s}\left(\lambda_{1}\right)\left(1-\vartheta\left(\lambda_{1}, n\right)\right) .
\end{gathered}
$$

For $V_{n}>c_{1}^{-1} U_{m_{0}}$, we obtain $t \leq \frac{U_{m_{0}}}{V_{n}}<c_{1} \leq c_{k}\left(t \in\left(0, \frac{U_{m_{0}}}{V_{n}}\right] ; k=1, \ldots, s\right)$ and

$$
\vartheta\left(\lambda_{1}, n\right)=\frac{\int_{0}^{\frac{U_{m_{0}}}{V_{n}}} t^{\lambda_{1}+\alpha-1} d t}{k_{s}\left(\lambda_{1}\right) \prod_{k=1}^{s} c_{k}^{\frac{\lambda+\alpha}{s}}}=\frac{\left(\lambda_{1}+\alpha\right)^{-1}}{k_{s}\left(\lambda_{1}\right) \prod_{k=1}^{s} c_{k}^{\frac{\lambda+\alpha}{s}}}\left(\frac{U_{m_{0}}}{V_{n}}\right)^{\lambda_{1}+\alpha} .
$$

Hence, we have (23).

For $b>0$, we find

$$
\begin{aligned}
\sum_{m=1}^{\infty} \frac{\mu_{m}}{U_{m}^{1+b}} & =\sum_{m=1}^{m_{0}} \frac{\mu_{m}}{U_{m}^{1+b}}+\sum_{m=m_{0}+1}^{\infty} \frac{\mu_{m}}{U_{m}^{1+b}} \\
& =\sum_{m=1}^{m_{0}} \frac{\mu_{m}}{U_{m}^{1+b}}+\sum_{m=m_{0}+1}^{\infty} \int_{m-1}^{m} \frac{U^{\prime}(x)}{U_{m}^{1+b}} d x \\
& <\sum_{m=1}^{m_{0}} \frac{\mu_{m}}{U_{m}^{1+b}}+\sum_{m=m_{0}+1}^{\infty} \int_{m-1}^{m} \frac{U^{\prime}(x)}{U^{1+b}(x)} d x \\
& =\sum_{m=1}^{m_{0}} \frac{\mu_{m}}{U_{m}^{1+b}}+\int_{m_{0}}^{\infty} \frac{d U(x)}{U^{1+b}(x)}=\sum_{m=1}^{m_{0}} \frac{\mu_{m}}{U_{m}^{1+b}}+\frac{1}{b U_{m_{0}}^{b}} \\
& =\frac{1}{b}\left(\frac{1}{U_{m}^{b}}+b \sum_{m=1}^{m_{0}} \frac{\mu_{m}}{U_{m}^{1+b}}\right) \\
\sum_{m=1}^{\infty} \frac{\mu_{m}}{U_{m}^{1+b}} & \geq \sum_{m=m_{0}}^{\infty} \frac{\mu_{m+1}}{U_{m}^{1+b}}=\sum_{m=m_{0}}^{\infty} \int_{m}^{m+1} \frac{U^{\prime}(x)}{U_{m}^{1+b}} d x \\
& >\sum_{m=m_{0}}^{\infty} \int_{m}^{m+1} \frac{U^{\prime}(x) d x}{U^{1+b}(x)}=\int_{m_{0}}^{\infty} \frac{d U(x)}{U^{1+b}(x)}=\frac{1}{b U_{m_{0}}^{b}}
\end{aligned}
$$

Hence we have (24). By the same way, we still have (25).

Note For example, $\mu_{m}=\frac{1}{m^{\sigma}}, v_{n}=\frac{1}{n^{\sigma}}(0 \leq \sigma \leq 1 ; m, n \in \mathbf{N})$ satisfy the conditions of Lemma $3\left(m_{0}=n_{0}=1\right)$.

\section{Main results and operator expressions}

Theorem 1 If $s \in \mathbf{N}, 0<c_{1} \leq \cdots \leq c_{s},-\alpha<\lambda_{1}, \lambda_{2} \leq 1-\alpha, \lambda_{1}+\lambda_{2}=\lambda, k_{s}\left(\lambda_{1}\right)$ is indicated by (11), then for $p>1,0<\|a\|_{p, \Phi_{\lambda}},\|b\|_{q, \Psi_{\lambda}}<\infty$, we have the following equivalent inequalities:

$$
I:=\sum_{n=1}^{\infty} \sum_{m=1}^{\infty} \prod_{k=1}^{s} \frac{\left(\min \left\{U_{m}, c_{k} V_{n}\right\}\right)^{\frac{\alpha}{s}} a_{m} b_{n}}{\left(\max \left\{U_{m}, c_{k} V_{n}\right\}\right)^{\frac{\lambda+\alpha}{s}}}<k_{s}\left(\lambda_{1}\right)\|a\|_{p, \Phi_{\lambda}}\|b\|_{q, \Psi_{\lambda}},
$$




$$
J:=\left\{\sum_{n=1}^{\infty} \frac{v_{n}}{V_{n}^{1-p \lambda_{2}}}\left[\sum_{m=1}^{\infty} \prod_{k=1}^{s} \frac{\left(\min \left\{U_{m}, c_{k} V_{n}\right\}\right)^{\frac{\alpha}{s}} a_{m}}{\left(\max \left\{U_{m}, c_{k} V_{n}\right\}\right)^{\frac{\lambda+\alpha}{s}}}\right]^{p}\right\}^{\frac{1}{p}}<k_{s}\left(\lambda_{1}\right)\|a\|_{p, \Phi_{\lambda}} .
$$

In particular, for $s=1$ (or $\left.c_{s}=\cdots=c_{1}\right)$, we have the following equivalent inequalities:

$$
\begin{aligned}
& \sum_{n=1}^{\infty} \sum_{m=1}^{\infty} \frac{\left(\min \left\{U_{m}, c_{1} V_{n}\right\}\right)^{\alpha} a_{m} b_{n}}{\left(\max \left\{U_{m}, c_{1} V_{n}\right\}\right)^{\lambda+\alpha}}<k_{1}\left(\lambda_{1}\right)\|a\|_{p, \Phi_{\lambda}}\|b\|_{q, \Psi_{\lambda}}, \\
& \left\{\sum_{n=1}^{\infty} \frac{v_{n}}{V_{n}^{1-p \lambda_{2}}}\left[\sum_{m=1}^{\infty} \frac{\left(\min \left\{U_{m}, c_{1} V_{n}\right\}\right)^{\alpha} a_{m}}{\left(\max \left\{U_{m}, c_{1} V_{n}\right\}\right)^{\lambda+\alpha}}\right]^{p}\right\}^{\frac{1}{p}}<k_{1}\left(\lambda_{1}\right)\|a\|_{p, \Phi_{\lambda}},
\end{aligned}
$$

where $k_{1}\left(\lambda_{1}\right)$ is indicated by (12).

Proof By Hölder's inequality with weight (cf. [29]), we have

$$
\begin{aligned}
{\left[\sum_{m=1}^{\infty}\right.} & \left.\prod_{k=1}^{s} \frac{\left(\min \left\{U_{m}, c_{k} V_{n}\right\}\right)^{\frac{\alpha}{s}}}{\left(\max \left\{U_{m}, c_{k} V_{n}\right\}\right)^{\frac{\lambda+\alpha}{s}}} a_{m}\right]^{p} \\
= & {\left[\sum_{m=1}^{\infty} \prod_{k=1}^{s} \frac{\left(\min \left\{U_{m}, c_{k} V_{n}\right\}\right)^{\frac{\alpha}{s}}}{\left(\max \left\{U_{m}, c_{k} V_{n}\right\}\right)^{\frac{\lambda+\alpha}{s}}}\left(\frac{U_{m}^{\frac{1-\lambda_{1}}{q}} a_{m}}{V_{n}^{\frac{1-\lambda_{2}}{p}} \mu_{m}^{\frac{1}{q}}}\right)\left(\frac{V_{n}^{\frac{1-\lambda_{2}}{p}} \mu_{m}^{\frac{1}{q}}}{U_{m}^{\frac{1-\lambda_{1}}{q}}}\right)\right]^{p} } \\
\leq & \sum_{m=1}^{\infty} \prod_{k=1}^{s} \frac{\left(\min \left\{U_{m}, c_{k} V_{n}\right\}\right)^{\frac{\alpha}{s}}}{\left(\max \left\{U_{m}, c_{k} V_{n}\right\}\right)^{\frac{\lambda+\alpha}{s}}}\left(\frac{U_{m}^{\left(1-\lambda_{1}\right) p / q}}{V_{n}^{1-\lambda_{2}} \mu_{m}^{p / q}} a_{m}^{p}\right) \\
& \times\left[\sum_{m=1}^{\infty} \prod_{k=1}^{s} \frac{\left(\min \left\{U_{m}, c_{k} V_{n}\right\}\right)^{\frac{\alpha}{s}}}{\left(\max \left\{U_{m}, c_{k} V_{n}\right\}\right)^{\frac{\lambda+\alpha}{s}}} \frac{V_{n}^{\left(1-\lambda_{2}\right)(q-1)}}{\mu_{m}}\right]_{m}^{p-1} \\
= & \frac{V_{n}^{1-p \lambda_{2}}}{\left(\varpi\left(\lambda_{1}, n\right)\right)^{1-p} v_{n}} \sum_{m=1}^{\infty} \prod_{k=1}^{s} \frac{\left(\min \left\{U_{m}, c_{k} V_{n}\right\}\right)^{\frac{\alpha}{s}}}{\left(\max \left\{U_{m}, c_{k} V_{n}\right\}\right)^{\frac{\lambda+\alpha}{s}}} \frac{U_{m}^{\left(1-\lambda_{1}\right)(p-1)} v_{n}}{V_{n}^{1-\lambda_{2}} \mu_{m}^{p-1}} a_{m}^{p} .
\end{aligned}
$$

In view of (20), we find

$$
\begin{aligned}
J & \leq\left(k_{s}\left(\lambda_{1}\right)\right)^{\frac{1}{q}}\left[\sum_{n=1}^{\infty} \sum_{m=1}^{\infty} \prod_{k=1}^{s} \frac{\left(\min \left\{U_{m}, c_{k} V_{n}\right\}\right)^{\frac{\alpha}{s}}}{\left(\max \left\{U_{m}, c_{k} V_{n}\right\}\right)^{\frac{\lambda+\alpha}{s}}} \frac{U_{m}^{\left(1-\lambda_{1}\right)(p-1)} v_{n}}{V_{n}^{1-\lambda_{2}} \mu_{m}^{p-1}} a_{m}^{p}\right]^{\frac{1}{p}} \\
& =\left(k_{s}\left(\lambda_{1}\right)\right)^{\frac{1}{q}}\left[\sum_{m=1}^{\infty} \sum_{n=1}^{\infty} \prod_{k=1}^{s} \frac{\left(\min \left\{U_{m}, c_{k} V_{n}\right\}\right)^{\frac{\alpha}{s}}}{\left(\max \left\{U_{m}, c_{k} V_{n}\right\}\right)^{\frac{\lambda+\alpha}{s}}} \frac{U_{m}^{\left(1-\lambda_{1}\right)(p-1)} v_{n}}{V_{n}^{1-\lambda_{2}} \mu_{m}^{p-1}} a_{m}^{p}\right]^{\frac{1}{p}} \\
& =\left(k_{s}\left(\lambda_{1}\right)\right)^{\frac{1}{q}}\left[\sum_{m=1}^{\infty} \omega\left(\lambda_{2}, m\right) \frac{U_{m}^{p\left(1-\lambda_{1}\right)-1}}{\mu_{m}^{p-1}} a_{m}^{p}\right]^{\frac{1}{p}} .
\end{aligned}
$$

Then, by (19), we have (27).

By Hölder's inequality ( $c f .[29])$, we have

$$
\begin{aligned}
I & =\sum_{n=1}^{\infty}\left[\frac{v_{n}^{\frac{1}{p}}}{V_{n}^{\frac{1}{p}-\lambda_{2}}} \sum_{m=1}^{\infty} \prod_{k=1}^{s} \frac{\left(\min \left\{U_{m}, c_{k} V_{n}\right\}\right)^{\frac{\alpha}{s}} a_{m}}{\left(\max \left\{U_{m}, c_{k} V_{n}\right\}\right)^{\frac{\lambda+\alpha}{s}}}\right]\left(\frac{V_{n}^{\frac{1}{p}-\lambda_{2}}}{v_{n}^{\frac{1}{p}}} b_{n}\right) \\
& \leq J\|b\|_{q, \Psi_{\lambda} .}
\end{aligned}
$$

Then, by (27), we have (26). 
On the other hand, assuming that (26) is valid, we set

$$
b_{n}:=\frac{v_{n}}{V_{n}^{1-p \lambda_{2}}}\left[\sum_{m=1}^{\infty} \prod_{k=1}^{s} \frac{\left(\min \left\{U_{m}, c_{k} V_{n}\right\}\right)^{\frac{\alpha}{s}} a_{m}}{\left(\max \left\{U_{m}, c_{k} V_{n}\right\}\right)^{\frac{\lambda+\alpha}{s}}}\right]^{p-1}, \quad n \in \mathbf{N}
$$

Then we find $J^{p}=\|b\|_{q, \Psi_{\lambda}}^{q}$. If $J=0$, then (27) is trivially valid; if $J=\infty$, then, by (31) and (19), it is impossible. Suppose that $0<J<\infty$. By (26), it follows that

$$
\begin{aligned}
&\|b\|_{q, \Psi_{\lambda}}^{q}=J^{p}=I<k_{s}\left(\lambda_{1}\right)\|a\|_{p, \Phi_{\lambda}}\|b\|_{q, \Psi_{\lambda}}, \\
&\|b\|_{q, \Psi_{\lambda}}^{q-1}=J<k_{s}\left(\lambda_{1}\right)\|a\|_{p, \Phi_{\lambda}},
\end{aligned}
$$

and then (27) follows, which is equivalent to (26).

Theorem 2 With the assumptions of Theorem 1 , if $m_{0}, n_{0} \in \mathbf{N}, \mu_{m} \geq \mu_{m+1}\left(m \in\left\{m_{0}, m_{0}+\right.\right.$ $1, \ldots\}), v_{n} \geq v_{n+1}\left(n \in\left\{n_{0}, n_{0}+1, \ldots\right\}\right), U(\infty)=V(\infty)=\infty$, then the constant factor $k_{s}\left(\lambda_{1}\right)$ in (26) and (27) is the best possible.

Proof For $\varepsilon \in\left(0, p\left(\lambda_{1}+\alpha\right)\right)$, we set $\tilde{\lambda}_{1}=\lambda_{1}-\frac{\varepsilon}{p}(\in(-\alpha, 1-\alpha)), \tilde{\lambda}_{2}=\lambda_{2}+\frac{\varepsilon}{p}(>-\alpha)$, and $\tilde{a}=\left\{\widetilde{a}_{m}\right\}_{m=1}^{\infty}, \widetilde{b}=\left\{\widetilde{b}_{n}\right\}_{n=1}^{\infty}$,

$$
\widetilde{a}_{m}:=U_{m}^{\widetilde{\lambda}_{1}-1} \mu_{m}=U_{m}^{\lambda_{1}-\frac{\varepsilon}{p}-1} \mu_{m}, \quad \widetilde{b}_{n}=V_{n}^{\widetilde{\lambda}_{2}-\varepsilon-1} v_{n}=V_{n}^{\lambda_{2}-\frac{\varepsilon}{q}-1} v_{n} .
$$

Then, by (24), (25) and (23), we have

$$
\begin{aligned}
\|\tilde{a}\|_{p, \Phi_{\lambda}}\|\widetilde{b}\|_{q, \Psi_{\lambda}}=\left(\sum_{m=1}^{\infty} \frac{\mu_{m}}{U_{m}^{1+\varepsilon}}\right)^{\frac{1}{p}}\left(\sum_{n=1}^{\infty} \frac{v_{n}}{V_{n}^{1+\varepsilon}}\right)^{\frac{1}{q}} \\
=\frac{1}{\varepsilon}\left(\frac{1}{U_{m_{0}}^{\varepsilon}}+\varepsilon O(1)\right)^{\frac{1}{p}}\left(\frac{1}{V_{n_{0}}^{\varepsilon}}+\varepsilon \widetilde{O}(1)\right)^{\frac{1}{q}}, \\
\widetilde{I}:=\sum_{n=1}^{\infty} \sum_{m=1}^{\infty} \prod_{k=1}^{s} \frac{\left(\min \left\{U_{m}, c_{k} V_{n}\right\}\right)^{\frac{\alpha}{s}}}{\left(\max \left\{U_{m}, c_{k} V_{n}\right\}\right)^{\frac{\lambda+\alpha}{s}}} \widetilde{a}_{m} \widetilde{b}_{n} \\
=\sum_{n=1}^{\infty}\left[\sum_{m=1}^{\infty} \prod_{k=1}^{s} \frac{\left(\min \left\{U_{m}, c_{k} V_{n}\right\}\right)^{\frac{\alpha}{s}}}{\left(\max \left\{U_{m}, c_{k} V_{n}\right\}\right)^{\frac{\lambda+\alpha}{s}}} \frac{V_{n}^{\tilde{\lambda}_{2}} \mu_{m}}{U_{m}^{1-\tilde{\lambda}_{1}}}\right] \frac{v_{n}}{V_{n}^{\varepsilon+1}} \\
=\sum_{n=1}^{\infty} \varpi\left(\tilde{\lambda}_{1}, n\right) \frac{v_{n}}{V_{n}^{\varepsilon+1}} \geq k_{s}\left(\tilde{\lambda}_{1}\right) \sum_{n=1}^{\infty}\left(1-\vartheta\left(\tilde{\lambda}_{1}, n\right)\right) \frac{v_{n}}{V_{n}^{\varepsilon+1}} \\
=k_{s}\left(\tilde{\lambda}_{1}\right)\left(\sum_{n=1}^{\infty} \frac{v_{n}}{V_{n}^{\varepsilon+1}}-\sum_{n=1}^{\infty} O\left(\frac{v_{n}}{V_{n}^{\frac{\varepsilon}{q}+\lambda_{1}+\alpha+1}}\right)\right) \\
=\frac{1}{\varepsilon} k_{s}\left(\tilde{\lambda}_{1}\right)\left[\frac{1}{V_{n}^{\varepsilon}}+\varepsilon(\widetilde{O}(1)-O(1))\right] .
\end{aligned}
$$

If there exists a positive constant $K \leq k_{s}\left(\lambda_{1}\right)$ such that (26) is valid when replacing $k_{s}\left(\lambda_{1}\right)$ with $K$, then, in particular, we have $\varepsilon \widetilde{I}<\varepsilon K\|\widetilde{a}\|_{p, \Phi_{\lambda}}\|\widetilde{b}\|_{q, \Psi_{\lambda}}$, namely

$$
k_{s}\left(\tilde{\lambda}_{1}\right)\left[\frac{1}{V_{n_{0}}^{\varepsilon}}+\varepsilon(\widetilde{O}(1)-O(1))\right]<K\left(\frac{1}{U_{m_{0}}^{\varepsilon}}+\varepsilon O(1)\right)^{\frac{1}{p}}\left(\frac{1}{V_{n_{0}}^{\varepsilon}}+\varepsilon \widetilde{O}(1)\right)^{\frac{1}{q}}
$$


It follows that $k_{s}\left(\lambda_{1}\right) \leq K\left(\varepsilon \rightarrow 0^{+}\right)$. Hence, $K=k_{s}\left(\lambda_{1}\right)$ is the best possible constant factor of (26).

The constant factor $k_{s}\left(\lambda_{1}\right)$ in (27) is still the best possible. Otherwise, we would reach a contradiction by (32) that the constant factor in (26) is not the best possible.

Remark 1 Inequality (26) is an extension of Hardy-Hilbert-type inequality (28) with parameters and a best possible constant factor.

For $p>1$, we find $\Psi_{\lambda}^{1-p}(n)=\frac{v_{n}}{V_{n}^{1-p \lambda_{2}}}$ and define the following normed spaces:

$$
\begin{aligned}
& l_{p, \Phi_{\lambda}}:=\left\{a=\left\{a_{m}\right\}_{m=1}^{\infty} ;\|a\|_{p, \Phi_{\lambda}}<\infty\right\}, \\
& l_{q, \Psi_{\lambda}}:=\left\{b=\left\{b_{n}\right\}_{n=1}^{\infty} ;\|b\|_{q, \Psi_{\lambda}}<\infty\right\}, \\
& l_{p, \Psi_{\lambda}^{1-p}}:=\left\{c=\left\{c_{n}\right\}_{n=1}^{\infty} ;\|c\|_{\left.p, \Psi_{\lambda}^{1-p}<\infty\right\} .}\right.
\end{aligned}
$$

Assuming that $a=\left\{a_{m}\right\}_{m=1}^{\infty} \in l_{p, \Phi_{\lambda}}$, setting

$$
c=\left\{c_{n}\right\}_{n=1}^{\infty}, \quad c_{n}:=\sum_{m=1}^{\infty} \prod_{k=1}^{s} \frac{\left(\min \left\{U_{m}, c_{k} V_{n}\right\}\right)^{\frac{\alpha}{s}}}{\left(\max \left\{U_{m}, c_{k} V_{n}\right\}\right)^{\frac{\lambda+\alpha}{s}}} a_{m}, \quad n \in \mathbf{N}
$$

we can rewrite (27) as follows:

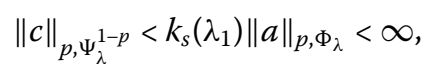

namely $c \in l_{p, \Psi_{\lambda}^{1-p}}$.

Definition 1 Define a Hardy-Hilbert-type operator $T: l_{p, \Phi_{\lambda}} \rightarrow l_{p, \Psi_{\lambda}^{1-p}}$ as follows: For any $a=\left\{a_{m}\right\}_{m=1}^{\infty} \in l_{p, \Phi_{\lambda}}$, there exists a unique representation $T a=c \in l_{p, \Psi_{\lambda}^{1-p}}$. Define the formal inner product of $T a$ and $b=\left\{b_{n}\right\}_{n=1}^{\infty} \in l_{q, \Psi_{\lambda}}$ as follows:

$$
(T a, b):=\sum_{n=1}^{\infty}\left[\sum_{m=1}^{\infty} \prod_{k=1}^{s} \frac{\left(\min \left\{U_{m}, c_{k} V_{n}\right\}\right)^{\frac{\alpha}{s}}}{\left(\max \left\{U_{m}, c_{k} V_{n}\right\}\right)^{\frac{\lambda+\alpha}{s}}} a_{m}\right] b_{n} .
$$

Then we can rewrite (26) and (27) as follows:

$$
\begin{aligned}
& (T a, b)<k_{s}\left(\lambda_{1}\right)\|a\|_{p, \Phi_{\lambda}}\|b\|_{q, \Psi_{\lambda}}, \\
& \|T a\|_{p, \Psi_{\lambda}^{1-p}}<k_{s}\left(\lambda_{1}\right)\|a\|_{p, \Phi_{\lambda}} .
\end{aligned}
$$

Define the norm of operator $T$ as follows:

$$
\|T\|:=\sup _{a(\neq \theta) \in l_{p, \Phi_{\lambda}}} \frac{\|T a\|_{p, \Psi_{\lambda}^{1-p}}}{\|a\|_{p, \Phi_{\lambda}}} .
$$

Then, by (36), we find $\|T\| \leq k_{s}\left(\lambda_{1}\right)$. Since by Theorem 2 the constant factor in (36) is the best possible, we have

$$
\|T\|=k_{s}\left(\lambda_{1}\right)
$$




\section{Some reverses}

In the following, we also set

$$
\begin{aligned}
& \widetilde{\Phi}_{\lambda}(m):=\left(1-\theta\left(\lambda_{2}, m\right)\right) \frac{U_{m}^{p\left(1-\lambda_{1}\right)-1}}{\mu_{m}^{p-1}}, \\
& \widetilde{\Psi}_{\lambda}(n):=\left(1-\vartheta\left(\lambda_{1}, n\right)\right) \frac{V_{n}^{q\left(1-\lambda_{2}\right)-1}}{v_{n}^{q-1}} \quad(m, n \in \mathbf{N}) .
\end{aligned}
$$

For $0<p<1$ or $p<0$, we still use the formal symbols of $\|a\|_{p, \Phi_{\lambda}},\|b\|_{q, \Psi_{\lambda}},\|a\|_{p, \widetilde{\Phi}_{\lambda}}$ and $\|b\|_{q, \widetilde{\Psi}_{\lambda}}$.

Theorem 3 If $s \in \mathbf{N}, 0<c_{1} \leq \cdots \leq c_{s},-\alpha<\lambda_{1}, \lambda_{2} \leq 1-\alpha, \lambda_{1}+\lambda_{2}=\lambda, k_{s}\left(\lambda_{1}\right)$ is indicated by (11), $m_{0}, n_{0} \in \mathbf{N}, \mu_{m} \geq \mu_{m+1}\left(m \in\left\{m_{0}, m_{0}+1, \ldots\right\}\right), v_{n} \geq v_{n+1}\left(n \in\left\{n_{0}, n_{0}+1, \ldots\right\}\right), U(\infty)=$ $V(\infty)=\infty$, then for $0<p<1,0<\|a\|_{p, \Phi_{\lambda}},\|b\|_{q, \Psi_{\lambda}}<\infty$, we have the following equivalent inequalities with the best possible constant factor $k_{s}\left(\lambda_{1}\right)$ :

$$
\begin{aligned}
I & =\sum_{n=1}^{\infty} \sum_{m=1}^{\infty} \prod_{k=1}^{s} \frac{\left(\min \left\{U_{m}, c_{k} V_{n}\right\}\right)^{\frac{\alpha}{s}} a_{m} b_{n}}{\left(\max \left\{U_{m}, c_{k} V_{n}\right\}\right)^{\frac{\lambda+\alpha}{s}}} \\
& >k_{s}\left(\lambda_{1}\right)\|a\|_{p, \widetilde{\Phi}_{\lambda}}\|b\|_{q, \Psi_{\lambda}}, \\
J & =\left\{\sum_{n=1}^{\infty} \frac{v_{n}}{V_{n}^{1-p \lambda_{2}}}\left[\sum_{m=1}^{\infty} \prod_{k=1}^{s} \frac{\left(\min \left\{U_{m}, c_{k} V_{n}\right\}\right)^{\frac{\alpha}{s}} a_{m}}{\left(\max \left\{U_{m}, c_{k} V_{n}\right\}\right)^{\frac{\lambda+\alpha}{s}}}\right]^{p}\right\}^{\frac{1}{p}} \\
& >k_{s}\left(\lambda_{1}\right)\|a\|_{p, \widetilde{\Phi}_{\lambda} .}
\end{aligned}
$$

Proof By the reverse Hölder's inequality (cf. [29]) and (20), we have the reverses of (30), (31) and (32). Then, by (22), we have (40). By (40) and the reverse of (32), we have (39).

On the other hand, assuming that (39) is valid, we set $b_{n}$ as in Theorem 1 . Then we find $J^{p}=\|b\|_{q, \Psi_{\lambda}}^{q}$. If $J=\infty$, then (40) is trivially valid; if $J=0$, then, by reverse of (31) and (22), it is impossible. Suppose that $0<J<\infty$. By (39), it follows that

$$
\begin{aligned}
& \|b\|_{q, \Psi_{\lambda}}^{q}=J^{p}=I>k_{s}\left(\lambda_{1}\right)\|a\|_{p, \widetilde{\Phi}_{\lambda}}\|b\|_{q, \Psi_{\lambda},}, \\
& \|b\|_{q, \Psi_{\lambda}}^{q-1}=J>k_{s}\left(\lambda_{1}\right)\|a\|_{p, \widetilde{\Phi}_{\lambda},}
\end{aligned}
$$

and then (40) follows, which is equivalent to (39).

For $\varepsilon \in\left(0, p\left(\lambda_{1}+\alpha\right)\right)$, we set $\widetilde{\lambda}_{1}, \widetilde{\lambda}_{2}, \widetilde{a}_{m}$ and $\widetilde{b}_{n}$ as (33). Then, by (24), (25) and (20), we find

$$
\begin{aligned}
\|a\|_{p, \widetilde{\Phi}_{\lambda}}\|b\|_{q, \Psi_{\lambda}} & =\left[\sum_{m=1}^{\infty}\left(1-\theta\left(\lambda_{2}, m\right)\right) \frac{\mu_{m}}{U_{m}^{1+\varepsilon}}\right]^{\frac{1}{p}}\left(\sum_{n=1}^{\infty} \frac{v_{n}}{V_{n}^{1+\varepsilon}}\right)^{\frac{1}{q}} \\
& =\left(\sum_{m=1}^{\infty} \frac{\mu_{m}}{U_{m}^{1+\varepsilon}}-\sum_{m=1}^{\infty} O\left(\frac{\mu_{m}}{U_{m}^{1+\lambda_{2}+\alpha+\varepsilon}}\right)\right)^{\frac{1}{p}}\left(\sum_{n=1}^{\infty} \frac{v_{n}}{V_{n}^{1+\varepsilon}}\right)^{\frac{1}{q}} \\
& =\frac{1}{\varepsilon}\left[\frac{1}{U_{m_{0}}^{\varepsilon}}+\varepsilon\left(O(1)-O_{1}(1)\right)\right]^{\frac{1}{p}}\left(\frac{1}{V_{n_{0}}^{\varepsilon}}+\varepsilon \widetilde{O}(1)\right)^{\frac{1}{q}},
\end{aligned}
$$




$$
\begin{aligned}
\widetilde{I} & =\sum_{n=1}^{\infty} \sum_{m=1}^{\infty} \prod_{k=1}^{s} \frac{\left(\min \left\{U_{m}, c_{k} V_{n}\right\}\right)^{\frac{\alpha}{s}}}{\left(\max \left\{U_{m}, c_{k} V_{n}\right\}\right)^{\frac{\lambda+\alpha}{s}}} \widetilde{a}_{m} \widetilde{b}_{n} \\
& =\sum_{n=1}^{\infty}\left[\sum_{m=1}^{\infty} \prod_{k=1}^{s} \frac{\left(\min \left\{U_{m}, c_{k} V_{n}\right\}\right)^{\frac{\alpha}{s}}}{\left(\max \left\{U_{m}, c_{k} V_{n}\right\}\right)^{\frac{\lambda+\alpha}{s}}} \frac{V_{n}^{\tilde{\lambda}_{2}} \mu_{m}}{U_{m}^{1-\tilde{\lambda}_{1}}}\right] \frac{v_{n}}{V_{n}^{\varepsilon+1}} \\
& =\sum_{n=1}^{\infty} \varpi\left(\widetilde{\lambda}_{1}, n\right) \frac{v_{n}}{V_{n}^{\varepsilon+1}} \leq k_{s}\left(\widetilde{\lambda}_{1}\right) \sum_{n=1}^{\infty} \frac{v_{n}}{V_{n}^{\varepsilon+1}} \\
& =\frac{1}{\varepsilon} k_{s}\left(\widetilde{\lambda}_{1}\right)\left(\frac{1}{V_{n_{0}}^{\varepsilon}}+\varepsilon \widetilde{O}(1)\right) .
\end{aligned}
$$

If there exists a constant $K \geq k_{s}\left(\lambda_{1}\right)$ such that (39) is valid when replacing $k_{s}\left(\lambda_{1}\right)$ with $K$, then, in particular, we have $\varepsilon \widetilde{I}>\varepsilon K\|\widetilde{a}\|_{p, \widetilde{\Phi}_{\lambda}}\|\widetilde{b}\|_{q, \Psi_{\lambda}}$, namely

$$
k_{s}\left(\widetilde{\lambda}_{1}\right)\left(\frac{1}{V_{n_{0}}^{\varepsilon}}+\varepsilon \widetilde{O}(1)\right)>K\left[\frac{1}{U_{m_{0}}^{\varepsilon}}+\varepsilon\left(O(1)-O_{1}(1)\right)\right]^{\frac{1}{p}}\left(\frac{1}{V_{n_{0}}^{\varepsilon}}+\varepsilon \widetilde{O}(1)\right)^{\frac{1}{q}} .
$$

It follows that $k_{s}\left(\lambda_{1}\right) \geq K\left(\varepsilon \rightarrow 0^{+}\right)$. Hence, $K=k_{s}\left(\lambda_{1}\right)$ is the best possible constant factor of (39).

The constant factor $k_{s}\left(\lambda_{1}\right)$ in (40) is still the best possible. Otherwise, we would reach a contradiction by the reverse of (32) that the constant factor in (39) is not the best possible.

Theorem 4 With the assumptions of Theorem 3, if $p<0$, then we have the following equivalent inequalities with the best possible constant factor $k_{s}\left(\lambda_{1}\right)$ :

$$
\begin{aligned}
I & =\sum_{n=1}^{\infty} \sum_{m=1}^{\infty} \prod_{k=1}^{s} \frac{\left(\min \left\{U_{m}, c_{k} V_{n}\right\}\right)^{\frac{\alpha}{s}} a_{m} b_{n}}{\left(\max \left\{U_{m}, c_{k} V_{n}\right\}\right)^{\frac{\lambda+\alpha}{s}}}>k_{s}\left(\lambda_{1}\right)\|a\|_{p, \Phi_{\lambda}}\|b\|_{q, \widetilde{\Psi}_{\lambda},} \\
J_{1} & :=\left\{\sum_{n=1}^{\infty} \frac{V_{n}^{p \lambda_{2}-1} v_{n}}{\left(1-\vartheta\left(\lambda_{1}, n\right)\right)^{p-1}}\left[\sum_{m=1}^{\infty} \prod_{k=1}^{s} \frac{\left(\min \left\{U_{m}, c_{k} V_{n}\right\}\right)^{\frac{\alpha}{s}} a_{m}}{\left(\max \left\{U_{m}, c_{k} V_{n}\right\}\right)^{\frac{\lambda+\alpha}{s}}}\right]^{p}\right\}^{\frac{1}{p}} \\
& >k_{s}\left(\lambda_{1}\right)\|a\|_{p, \Phi_{\lambda} .}
\end{aligned}
$$

Proof By the reverse Hölder's inequality with weight (cf. [29]), since $p<0$, by (23), we have

$$
\begin{aligned}
& {\left[\sum_{m=1}^{\infty} \prod_{k=1}^{s} \frac{\left(\min \left\{U_{m}, c_{k} V_{n}\right\}\right)^{\frac{\alpha}{s}} a_{m}}{\left(\max \left\{U_{m}, c_{k} V_{n}\right\}\right)^{\frac{\lambda+\alpha}{s}}}\right]^{p}} \\
& =\left[\sum_{m=1}^{\infty} \prod_{k=1}^{s} \frac{\left(\min \left\{U_{m}, c_{k} V_{n}\right\}\right)^{\frac{\alpha}{s}}}{\left(\max \left\{U_{m}, c_{k} V_{n}\right\}\right)^{\frac{\lambda+\alpha}{s}}}\left(\frac{U_{m}^{\left(1-\lambda_{1}\right) / q}}{V_{n}^{\left(1-\lambda_{2}\right) / p} \mu_{m}^{1 / q}} a_{m}\right)\left(\frac{V_{n}^{\left(1-\lambda_{2}\right) / p} \mu_{m}^{1 / q}}{U_{m}^{\left(1-\lambda_{1}\right) / q}}\right)\right]^{p} \\
& \leq \sum_{m=1}^{\infty} \prod_{k=1}^{s} \frac{\left(\min \left\{U_{m}, c_{k} V_{n}\right\}\right)^{\frac{\alpha}{s}}}{\left(\max \left\{U_{m}, c_{k} V_{n}\right\}\right)^{\frac{\lambda+\alpha}{s}}} \frac{U_{m}^{\left(1-\lambda_{1}\right) p / q}}{V_{n}^{1-\lambda_{2}} \mu_{m}^{p / q}} a_{m}^{p} \\
& \quad \times\left[\sum_{m=1}^{\infty} \prod_{k=1}^{s} \frac{\left(\min \left\{U_{m}, c_{k} V_{n}\right\}\right)^{\frac{\alpha}{s}}}{\left.\left(\max \left\{U_{m}, c_{k} V_{n}\right\}\right)^{\frac{\lambda+\alpha}{s}} \frac{V_{n}^{\left(1-\lambda_{2}\right)(q-1)}}{U_{m}}\right]_{m}^{p-1}}\right]^{p-\lambda_{1}} \\
& =\frac{V_{n}^{1-p \lambda_{2}}}{\left(\varpi\left(\lambda_{1}, n\right)\right)^{1-p}} \sum_{m=1}^{\infty} \prod_{k=1}^{s} \frac{\left(\min \left\{U_{m}, c_{k} V_{n}\right\}\right)^{\frac{\alpha}{s}}}{\left(\max \left\{U_{m}, c_{k} V_{n}\right\}\right)^{\frac{\lambda+\alpha}{s}} \frac{U_{m}^{\left(1-\lambda_{1}\right)(p-1)}}{V_{n}^{1-\lambda_{2}} \mu_{m}^{p-1}} a_{m}^{p}}
\end{aligned}
$$




$$
\begin{aligned}
& \leq \frac{\left(k_{s}\left(\lambda_{1}\right)\right)^{p-1} V_{n}^{1-p \lambda_{2}}}{\left(1-\vartheta\left(\lambda_{1}, n\right)\right)^{1-p} v_{n}} \sum_{m=1}^{\infty} \prod_{k=1}^{s} \frac{\left(\min \left\{U_{m}, c_{k} V_{n}\right\}\right)^{\frac{\alpha}{s}}}{\left(\max \left\{U_{m}, c_{k} V_{n}\right\}\right)^{\frac{\lambda+\alpha}{s}}} \frac{U_{m}^{\left(1-\lambda_{1}\right)(p-1)} v_{n}}{V_{n}^{1-\lambda_{2}} \mu_{m}^{p-1}} a_{m}^{p}, \\
& J_{1} \geq\left(k_{s}\left(\lambda_{1}\right)\right)^{\frac{1}{q}}\left\{\sum_{n=1}^{\infty} \sum_{m=1}^{\infty} \prod_{k=1}^{s} \frac{\left(\min \left\{U_{m}, c_{k} V_{n}\right\}\right)^{\frac{\alpha}{s}}}{\left(\max \left\{U_{m}, c_{k} V_{n}\right\}\right)^{\frac{\lambda+\alpha}{s}}} \frac{U_{m}^{\left(1-\lambda_{1}\right)(p-1)} v_{n}}{V_{n}^{1-\lambda_{2}} \mu_{m}^{p-1}} a_{m}^{p}\right\}^{\frac{1}{p}} \\
&=\left(k_{s}\left(\lambda_{1}\right)\right)^{\frac{1}{q}}\left\{\sum_{m=1}^{\infty} \sum_{n=1}^{\infty} \prod_{k=1}^{s} \frac{\left(\min \left\{U_{m}, c_{k} V_{n}\right\}\right)^{\frac{\alpha}{s}}}{\left(\max \left\{U_{m}, c_{k} V_{n}\right\}\right)^{\frac{\lambda+\alpha}{s}}} \frac{U_{m}^{\left(1-\lambda_{1}\right)(p-1)} v_{n}}{V_{n}^{1-\lambda_{2}} \mu_{m}^{p-1}} a_{m}^{p}\right\}^{\frac{1}{p}} \\
&=\left(k_{s}\left(\lambda_{1}\right)\right)^{\frac{1}{q}}\left\{\sum_{m=1}^{\infty} \omega\left(\lambda_{2}, m\right) \frac{U_{m}^{p\left(1-\lambda_{1}\right)-1}}{\mu_{m}^{p-1}} a_{m}^{p}\right\}^{\frac{1}{p}} .
\end{aligned}
$$

Then, by (19), we have (44).

By the reverse Hölder's inequality ( $c f$. [29]), we have

$$
\begin{aligned}
I & =\sum_{n=1}^{\infty} \frac{V_{n}^{\lambda_{2}-\frac{1}{p}} v_{n}^{1 / p}}{\left(1-\vartheta\left(\lambda_{1}, n\right)\right)^{1 / q}}\left[\sum_{m=1}^{\infty} \prod_{k=1}^{s} \frac{\left(\min \left\{U_{m}, c_{k} V_{n}\right\}\right)^{\frac{\alpha}{s}} a_{m}}{\left(\max \left\{U_{m}, c_{k} V_{n}\right\}\right)^{\frac{\lambda+\alpha}{s}}}\right]\left[\left(1-\vartheta\left(\lambda_{1}, n\right)\right)^{\frac{1}{q}} \frac{V_{n}^{\frac{1}{p}-\lambda_{2}}}{v_{n}^{1 / p}} b_{n}\right] \\
& \geq J_{1}\|b\|_{q, \widetilde{\Psi}_{\lambda} .}
\end{aligned}
$$

Then, by (42), we have (41).

On the other hand, assuming that (41) is valid, we set $b_{n}$ as follows:

$$
b_{n}:=\frac{V_{n}^{p \lambda_{2}-1} v_{n}}{\left(1-\vartheta\left(\lambda_{1}, n\right)\right)^{p-1}}\left[\sum_{m=1}^{\infty} \prod_{k=1}^{s} \frac{\left(\min \left\{U_{m}, c_{k} V_{n}\right\}\right)^{\frac{\alpha}{s}} a_{m}}{\left(\max \left\{U_{m}, c_{k} V_{n}\right\}\right)^{\frac{\lambda+\alpha}{s}}}\right]^{p-1}, \quad n \in \mathbf{N}
$$

Then we find $J_{1}^{p}=\|b\|_{q, \widetilde{\Psi}_{\lambda}}^{q}$. If $J_{1}=\infty$, then (42) is trivially valid; if $J_{1}=0$, then by (43) and (19) it is impossible. Suppose that $0<J_{1}<\infty$. By (41), it follows that

$$
\begin{aligned}
& \|b\|_{q, \widetilde{\Psi}_{\lambda}}^{q}=J_{1}^{p}=I>k_{s}\left(\lambda_{1}\right)\|a\|_{p, \Phi_{\lambda}}\|b\|_{q, \widetilde{\Psi}_{\lambda}}, \\
& \|b\|_{q, \widetilde{\Psi}_{\lambda}}^{q-1}=J_{1}>k_{s}\left(\lambda_{1}\right)\|a\|_{p, \Phi_{\lambda}},
\end{aligned}
$$

and then (42) follows, which is equivalent to (41).

For $\varepsilon \in\left(0, q\left(\lambda_{2}+\alpha\right)\right)$, we set $\tilde{\lambda}_{1}=\lambda_{1}+\frac{\varepsilon}{q}(>-\alpha), \tilde{\lambda}_{2}=\lambda_{2}-\frac{\varepsilon}{q}(\in(-\alpha, 1-\alpha))$, and

$$
\widetilde{a}_{m}:=U_{m}^{\tilde{\lambda}_{1}-1-\varepsilon} \mu_{m}=U_{m}^{\lambda_{1}-\frac{\varepsilon}{p}-1} \mu_{m}, \quad \widetilde{b}_{n}=V_{n}^{\tilde{\lambda}_{2}-1} v_{n}=V_{n}^{\lambda_{2}-\frac{\varepsilon}{q}-1} v_{n}
$$

Then, by (24), (25) and (19), we have

$$
\begin{aligned}
\|\widetilde{a}\|_{p, \Phi_{\lambda}}\|\widetilde{b}\|_{q, \widetilde{\Psi}_{\lambda}} & =\left(\sum_{m=1}^{\infty} \frac{\mu_{m}}{U_{m}^{1+\varepsilon}}\right)^{\frac{1}{p}}\left[\sum_{n=1}^{\infty}\left(1-\vartheta\left(\lambda_{1}, n\right)\right) \frac{v_{n}}{V_{n}^{1+\varepsilon}}\right]^{\frac{1}{q}} \\
& =\left(\sum_{m=1}^{\infty} \frac{\mu_{m}}{U_{m}^{1+\varepsilon}}\right)^{\frac{1}{p}}\left(\sum_{n=1}^{\infty} \frac{v_{n}}{V_{n}^{1+\varepsilon}}-\sum_{n=1}^{\infty} O\left(\frac{v_{n}}{V_{n}^{1+\lambda_{1}+\alpha+\varepsilon}}\right)\right)^{\frac{1}{q}} \\
& =\frac{1}{\varepsilon}\left(\frac{1}{U_{m_{0}}^{\varepsilon}}+\varepsilon O(1)\right)^{\frac{1}{p}}\left[\frac{1}{V_{n_{0}}^{\varepsilon}}+\varepsilon\left(\widetilde{O}(1)-O_{1}(1)\right)\right]^{\frac{1}{q}},
\end{aligned}
$$




$$
\begin{aligned}
\widetilde{I} & =\sum_{n=1}^{\infty} \sum_{m=1}^{\infty} \prod_{k=1}^{s} \frac{\left(\min \left\{U_{m}, c_{k} V_{n}\right\}\right)^{\frac{\alpha}{s}}}{\left(\max \left\{U_{m}, c_{k} V_{n}\right\}\right)^{\frac{\lambda+\alpha}{s}}} \widetilde{a}_{m} \widetilde{b}_{n} \\
& =\sum_{m=1}^{\infty}\left[\sum_{n=1}^{\infty} \prod_{k=1}^{s} \frac{\left(\min \left\{U_{m}, c_{k} V_{n}\right\}\right)^{\frac{\alpha}{s}}}{\left(\max \left\{U_{m}, c_{k} V_{n}\right\}\right)^{\frac{\lambda+\alpha}{s}}} \frac{U_{m}^{\widetilde{\lambda}_{1}} v_{n}}{V_{n}^{1-\tilde{\lambda}_{2}}}\right] \frac{\mu_{m}}{U_{m}^{1+\varepsilon}} \\
& =\sum_{m=1}^{\infty} \omega\left(\widetilde{\lambda}_{2}, m\right) \frac{\mu_{m}}{U_{m}^{1+\varepsilon}} \leq k_{s}\left(\widetilde{\lambda}_{1}\right) \sum_{n=1}^{\infty} \frac{\mu_{m}}{U_{m}^{1+\varepsilon}} \\
& =\frac{1}{\varepsilon} k_{s}\left(\widetilde{\lambda}_{1}\right)\left(\frac{1}{U_{m}^{\varepsilon}}+\varepsilon O(1)\right) .
\end{aligned}
$$

If there exists a constant $K \geq k_{s}\left(\lambda_{1}\right)$ such that (41) is valid when replacing $k_{s}\left(\lambda_{1}\right)$ with $K$, then, in particular, we have $\varepsilon \widetilde{I}>\varepsilon K\|\widetilde{a}\|_{p, \Phi_{\lambda}}\|\widetilde{b}\|_{q, \widetilde{\Psi}_{\lambda}}$, namely

$$
\begin{aligned}
& k_{s}\left(\tilde{\lambda}_{1}\right)\left(\frac{1}{U_{m_{0}}^{\varepsilon}}+\varepsilon O(1)\right) \\
& \quad>K\left(\frac{1}{U_{m_{0}}^{\varepsilon}}+\varepsilon O(1)\right)^{\frac{1}{p}}\left[\frac{1}{V_{n_{0}}^{\varepsilon}}+\varepsilon\left(\widetilde{O}(1)-O_{1}(1)\right)\right]^{\frac{1}{q}} .
\end{aligned}
$$

It follows that $k_{s}\left(\lambda_{1}\right) \geq K\left(\varepsilon \rightarrow 0^{+}\right)$. Hence, $K=k_{s}\left(\lambda_{1}\right)$ is the best possible constant factor of (41).

The constant factor $k_{s}\left(\lambda_{1}\right)$ in (42) is still the best possible. Otherwise, we would reach a contradiction by (44) that the constant factor in (41) is not the best possible.

Remark 2 (i) For $\alpha=0,0<\lambda_{1}, \lambda_{2} \leq 1$ in (26) and (27), we have the following equivalent inequalities:

$$
\begin{aligned}
& \sum_{n=1}^{\infty} \sum_{m=1}^{\infty} \frac{a_{m} b_{n}}{\prod_{k=1}^{s}\left(\max \left\{U_{m}, c_{k} V_{n}\right\}\right)^{\frac{\lambda}{s}}}<\widetilde{k}_{s}\left(\lambda_{1}\right)\|a\|_{p, \Phi_{\lambda}}\|b\|_{q, \Psi_{\lambda},} \\
& \left\{\sum_{n=1}^{\infty} \frac{v_{n}}{V_{n}^{1-p \lambda_{2}}}\left[\sum_{m=1}^{\infty} \frac{a_{m}}{\prod_{k=1}^{s}\left(\max \left\{U_{m}, c_{k} V_{n}\right\}\right)^{\frac{\lambda}{s}}}\right]^{p}\right\}^{\frac{1}{p}}<\widetilde{k}_{s}\left(\lambda_{1}\right)\|a\|_{p, \Phi_{\lambda}},
\end{aligned}
$$

where $\widetilde{k}_{s}\left(\lambda_{1}\right)$ is indicated by (14);

(ii) for $\alpha=-\lambda,-1 \leq \lambda_{1}, \lambda_{2}<0$ in (26) and (27), we have the following equivalent inequalities:

$$
\begin{aligned}
& \sum_{n=1}^{\infty} \sum_{m=1}^{\infty} \frac{a_{m} b_{n}}{\prod_{k=1}^{s}\left(\min \left\{U_{m}, c_{k} V_{n}\right\}\right)^{\frac{\lambda}{s}}}<\widehat{k}_{s}\left(\lambda_{1}\right)\|a\|_{p, \Phi_{\lambda}}\|b\|_{q, \Psi_{\lambda}}, \\
& \left\{\sum_{n=1}^{\infty} \frac{v_{n}}{V_{n}^{1-p \lambda_{2}}}\left[\sum_{m=1}^{\infty} \frac{a_{m}}{\prod_{k=1}^{s}\left(\min \left\{U_{m}, c_{k} V_{n}\right\}\right)^{\frac{\lambda}{s}}}\right]^{p}\right\}^{\frac{1}{p}}<\widehat{k}_{s}\left(\lambda_{1}\right)\|a\|_{p, \Phi_{\lambda}},
\end{aligned}
$$

where $\widehat{k}_{s}\left(\lambda_{1}\right)$ is indicated by $(15)$;

(iii) for $\lambda=0, \lambda_{2}=-\lambda_{1}$, in (26) and (27), we have the following equivalent inequalities:

$$
\sum_{n=1}^{\infty} \sum_{m=1}^{\infty} \prod_{k=1}^{s}\left(\frac{\min \left\{U_{m}, c_{k} V_{n}\right\}}{\max \left\{U_{m}, c_{k} V_{n}\right\}}\right)^{\frac{\alpha}{s}} a_{m} b_{n}<k_{s}^{(0)}\left(\lambda_{1}\right)\|a\|_{p, \Phi_{\lambda}}\|b\|_{q, \Psi_{\lambda}},
$$




$$
\left\{\sum_{n=1}^{\infty} \frac{v_{n}}{V_{n}^{1+p \lambda_{1}}}\left[\sum_{m=1}^{\infty} \prod_{k=1}^{s}\left(\frac{\min \left\{U_{m}, c_{k} V_{n}\right\}}{\max \left\{U_{m}, c_{k} V_{n}\right\}}\right)^{\frac{\alpha}{s}} a_{m}\right]^{p}\right\}^{\frac{1}{p}}<k_{s}^{(0)}\left(\lambda_{1}\right)\|a\|_{p, \Phi_{\lambda}}
$$

where $k_{s}^{(0)}\left(\lambda_{1}\right)$ is indicated by (16) $\left(\left|\lambda_{1}\right|<\alpha, 0<\alpha \leq \frac{1}{2} ;\left|\lambda_{1}\right|<1-\alpha, \frac{1}{2}<\alpha \leq 1\right)$.

By Theorem 2, the constant factors in the above inequalities are all the best possible. We still can obtain some particular reverse inequalities with the best possible constant factors by Theorem 3 and Theorem 4 .

\section{Competing interests}

The authors declare that they have no competing interests.

\section{Authors' contributions}

BY carried out the mathematical studies, participated in the sequence alignment and drafted the manuscript. QC participated in the design of the study and performed the numerical analysis. All authors read and approved the final manuscript.

\section{Author details}

'Department of Mathematics, Guangdong University of Education, Guangzhou, Guangdong 510303, P.R. China.

${ }^{2}$ Department of Computer Science, Guangdong University of Education, Guangzhou, Guangdong 510303, P.R. China.

\section{Acknowledgements}

This work is supported by the National Natural Science Foundation of China (No. 61370186), and the Science and Technology Planning Project of Guangzhou (No. 2014J4100032, No. 201510010203). We are grateful for their help.

Received: 6 May 2015 Accepted: 24 September 2015 Published online: 24 October 2015

\section{References}

1. Hardy, GH, Littlewood, JE, Pólya, G: Inequalities. Cambridge University Press, Cambridge (1934)

2. Mitrinović, DS, Pečarić, JE, Fink, AM: Inequalities Involving Functions and Their Integrals and Derivatives. Kluwer Academic, Boston (1991)

3. Yang, BC: Hilbert-Type Integral Inequalities. Bentham Science Publishers Ltd., Sharjah (2009)

4. Yang, BC: Discrete Hilbert-Type Inequalities. Bentham Science Publishers Ltd., Sharjah (2011)

5. Yang, BC: The Norm of Operator and Hilbert-Type Inequalities. Science Press, Beijing (2009)

6. Yang, BC: Two Types of Multiple Half-Discrete Hilbert-Type Inequalities. Lambert Academic Publishing, Saarbrücken (2012)

7. Persson, LE, et al.: Commutators of Hardy operators in vanishing Morrey spaces. In: 9th International Conference on Mathematical Problems in Engineering, Aerospace and Sciences (ICNPAA 2012). AIP Conference Proceedings, vol. 1493, pp. 859-866 (2012). doi:10.1063/1.4765588

8. Yang, BC: On Hilbert's integral inequality. J. Math. Anal. Appl. 220, 778-785 (1998)

9. Yang, BC, Brnetić, I, Krnić, M, Pečarić, JE: Generalization of Hilbert and Hardy-Hilbert integral inequalities. Math. Inequal. Appl. 8(2), 259-272 (2005)

10. Krnić, M, Pečarić, JE: Hilbert's inequalities and their reverses. Publ. Math. (Debr.) 67(3-4), 315-331 (2005)

11. Yang, BC, Rassias, TM: On the way of weight coefficient and research for Hilbert-type inequalities. Math. Inequal. Appl. 6(4), 625-658 (2003)

12. Yang, BC, Rassias, TM: On a Hilbert-type integral inequality in the subinterval and its operator expression. Banach J. Math. Anal. 4(2), 100-110 (2010)

13. Azar, L: On some extensions of Hardy-Hilbert's inequality and applications. J. Inequal. Appl. 2009, Article ID 546829 (2009)

14. Arpad, B, Choonghong, O: Best constant for certain multilinear integral operator. J. Inequal. Appl. 2006, Article ID $28582(2006)$

15. Kuang, JC, Debnath, L: On Hilbert's type inequalities on the weighted Orlicz spaces. Pac. J. Appl. Math. 1(1), 95-103 (2007)

16. Zhong, WY: The Hilbert-type integral inequality with a homogeneous kernel of Lambda-degree. J. Inequal. Appl. 2008, Article ID 917392 (2008)

17. Hong, Y: On Hardy-Hilbert integral inequalities with some parameters. J. Inequal. Pure Appl. Math. 6(4), 92 (2005)

18. Zhong, WY, Yang, BC: On multiple Hardy-Hilbert's integral inequality with kernel. J. Inequal. Appl. 2007, Article ID 27962 (2007). doi:10.1155/2007/27962

19. Yang, BC, Krnić, M: On the norm of a multi-dimensional Hilbert-type operator. Sarajevo J. Math. 7(20), 223-243 (2011)

20. Krnić, M, Pečarić, JE, Vuković, P: On some higher-dimensional Hilbert's and Hardy-Hilbert's type integral inequalities with parameters. Math. Inequal. Appl. 11, 701-716 (2008)

21. Krnić, M, Vuković, P: On a multidimensional version of the Hilbert-type inequality. Anal. Math. 38, 291-303 (2012)

22. Rassias, TM, Yang, BC: On half-discrete Hilbert's inequality. Appl. Math. Comput. 220, 75-93 (2013)

23. Rassias, TM, Yang, BC: A multidimensional half-discrete Hilbert-type inequality and the Riemann zeta function. Appl. Math. Comput. 225, 263-277 (2013)

24. Rassias, TM, Yang, BC: On a multidimensional half - discrete Hilbert - type inequality related to the hyperbolic cotangent function. Appl. Math. Comput. 242, 800-813 (2014) 
25. Rassias, TM, Yang, BC: On a multidimensional Hilbert-type integral inequality associated to the gamma function. Appl. Math. Comput. 249, 408-418 (2014)

26. Li, YJ, He, B: On inequalities of Hilbert's type. Bull. Aust. Math. Soc. 76(1), 1-13 (2007)

27. Yang, BC: On a more accurate multidimensional Hilbert-type inequality with parameters. Math. Inequal. Appl. 18(2), 429-441 (2015)

28. Yang, BC: An extension of a Hardy-Hilbert-type inequality. J. Guangdong Univ. Educ. 35(3), 1-8 (2015)

29. Kuang, JC: Applied Inequalities. Shangdong Science Technic Press, Jinan (2004)

Submit your manuscript to a SpringerOpen ${ }^{\circ}$ journal and benefit from:

- Convenient online submission

- Rigorous peer review

- Immediate publication on acceptance

Open access: articles freely available online

- High visibility within the field

- Retaining the copyright to your article 